\title{
Bayesian Robust Principal Component Analysis
}

\author{
${ }^{1,2}$ Xinghao Ding, ${ }^{1}$ Lihan He and ${ }^{1}$ Lawrence Carin \\ ${ }^{1}$ Department of Electrical \& Computer Engineering \\ Duke University \\ Durham, NC 27708-0291, USA \\ ${ }^{2}$ Department of Communication Engineering \\ Xiamen University \\ Xiamen, Fujian, 361005, China \\ Email:xd11@duke.edu, lihan@ece.duke.edu, lcarin@ece.duke.edu
}

\begin{abstract}
A hierarchical Bayesian model is considered for decomposing a matrix into low-rank and sparse components, assuming the observed matrix is a superposition of the two. The matrix is assumed noisy, with unknown and possibly non-stationary noise statistics. The Bayesian framework infers an approximate representation for the noise statistics while simultaneously inferring the low-rank and sparse-outlier contributions; the model is robust to a broad range of noise levels, without having to change model hyperparameter settings. In addition, the Bayesian framework allows exploitation of additional structure in the matrix. For example, in video applications each row (or column) corresponds to a video frame, and we introduce a Markov dependency between consecutive rows in the matrix (corresponding to consecutive frames in the video). The properties of this Markov process are also inferred based on the observed matrix, while simultaneously denoising and recovering the low-rank and sparse components. We compare the Bayesian model to a state-of-the-art optimization-based implementation of robust PCA; considering several examples, we demonstrate competitive performance of the proposed model.
\end{abstract}

Index Terms

Bayesian modeling, principal component analysis, low-rank matrix, sparsity, Markov dependency

\section{INTRODUCTION}

Natural high-dimensional data, such as images, video, documents, biological data, and socialnetwork data often reside in a low-dimensional subspace [1] or low-dimensional manifold [2], [3]. 
For example, the frames in a video sequence typically reside in a subspace of dimension much smaller than that of the number of frame pixels, due to strong correlation between consecutive video frames. Movie rating data constitute another example, among which the Netflix Prize [4] is perhaps the most famous example. The rating matrix is typically low-rank, because of similarities among movies and among users/people.

Principal component analysis (PCA) [5], [6], computed via a singular value decomposition (SVD), is a common tool for extracting low-dimensional information from the aforementioned high-dimensional data. Utilizing SVD, a data matrix $\boldsymbol{L} \in \mathbb{R}^{N \times M}$ with rank $r$ can be decomposed as

$$
\boldsymbol{L}=\boldsymbol{D} \boldsymbol{\Lambda} \boldsymbol{W}^{T}=\sum_{i=1}^{r} \lambda_{i} \boldsymbol{d}_{i} \boldsymbol{w}_{i}^{T}
$$

where diagonal matrix $\boldsymbol{\Lambda}=\operatorname{diag}\left(\lambda_{1}, \ldots, \lambda_{r}\right) \in \mathbb{R}^{r \times r}$ consists of singular values of $\boldsymbol{L}, \boldsymbol{D}=$ $\left[\boldsymbol{d}_{1}, \ldots, \boldsymbol{d}_{r}\right] \in \mathbb{R}^{N \times r}$ and $\boldsymbol{W}=\left[\boldsymbol{w}_{1}, \ldots, \boldsymbol{w}_{r}\right] \in \mathbb{R}^{M \times r}$ are the matrices of left- and right-singular vectors, respectively. If $\boldsymbol{L}$ is low-rank, $r \ll \min (N, M)$.

Low-rank matrix representations have recently received significant attention. In these studies two types of noise models are often considered. One class corresponds to generally smallmagnitude perturbations to all matrix elements [7], [8] (e.g., i.i.d. Gaussian noise added to each matrix element). If the noise energy is small relative to the dominant singular values of the SVD, this noise class often manifests relatively modest changes to a noise-free PCA analysis. Specifically, it often yields small perturbations to the singular values, but does not significantly impact the principal vectors, and shrinkage methods may be employed for denoising [9]. The second noise class is sparse and of arbitrary amplitude, it impacting a small subset of the matrix elements [10], [11]. For example, a moving object in video, in the presence of a static background, manifests such sparse noise (if the matrix has rows/columns defined by the video frames). In this situation the sparse outliers may easily corrupt classical PCA, causing the singular vectors and singular values to change dramatically.

Many algorithms have been proposed to address the brittleness of classical PCA with respect to outlier (sparse) noise, yielding the field of robust PCA [12], [13], [14], [15], [16]. However, these previous methods are typically computationally inefficient, and they do not possess the strong performance guarantees provided by very recent work [10], [17]. In particular, in [10], [17], [11] the authors have proposed convex optimization to address the robust PCA problem. 
The observation matrix is assumed represented as $\boldsymbol{Y}=\boldsymbol{L}+\boldsymbol{S}$, where $\boldsymbol{L}$ is a low-rank matrix and $S$ is a sparse matrix with a small fraction of (arbitrary magnitude) nonzero entries. This research seeks to solve for $\boldsymbol{L}$ with the following optimization problem:

$$
\min _{\boldsymbol{L}, \boldsymbol{S}}\|\boldsymbol{L}\|_{*}+\zeta\|\boldsymbol{S}\|_{1} \quad \text { s.t. } \quad \boldsymbol{L}+\boldsymbol{S}=\boldsymbol{Y}
$$

where $\|\cdot\|_{*}$ and $\|\cdot\|_{1}$ denote the matrix nuclear norm (sum of singular values) and $\ell_{1}$ norm, respectively, and $\zeta>0$ is a parameter to balance the two terms. Candés et al. showed in [17] that under broad conditions the proposed convex optimization method exactly recovers $\boldsymbol{L}$ and $S$, and the results in [10], [17] demonstrated encouraging performance on real data.

In practice, measurement noise (of the first type discussed above) exists everywhere within the matrix and sometimes this dense (distributed) noise contribution cannot be ignored. The problem of additive noise of this type, denoted $\boldsymbol{E}$, is discussed in [10], [17], with this addressed explicitly in [18]. The work in [18] modified (2) to include an error term, i.e., $\min _{\boldsymbol{L}, \boldsymbol{S}}\|\boldsymbol{L}\|_{*}+$ $\zeta\|\boldsymbol{S}\|_{1}+(2 \mu)^{-1}\|\boldsymbol{Y}-\boldsymbol{L}-\boldsymbol{S}\|_{F}^{2}$, with $\|\cdot\|_{F}$ denoting the Frobenius norm. However, to choose the balance parameter $\mu$, the noise variance has to be known.

In this paper we also consider the observation matrix $\boldsymbol{Y}=\boldsymbol{L}+\boldsymbol{S}+\boldsymbol{E}$, with the presence of both sparse noise $\boldsymbol{S}$ and the dense noise $\boldsymbol{E}$. Although $\boldsymbol{E}$ is usually not desired and is discarded, the sparse outliers $S$ are sometimes of interest (e.g., for tracking moving entities in video). Therefore, in the remainder of the paper we do not regard the sparse outliers as noise; we call $\boldsymbol{L}, \boldsymbol{S}$ and $\boldsymbol{E}$ the low-rank, sparse, and noise components, respectively. In the proposed Bayesian framework, the noise statistics of $\boldsymbol{E}$ are learned (approximately) based on the data, simultaneous with the learning of $\boldsymbol{L}$ and $\boldsymbol{S}$. When presenting results, we demonstrate that the proposed Bayesian model is robust to a broad range of noise variance (and to nonstationary noise), without having to change model hyperparameters.

The Bayesian model infers approximations to full posterior distributions on all model parameters, and therefore we obtain approximate probability distributions for $\boldsymbol{L}, \boldsymbol{S}$ and $\boldsymbol{E}$. One may compute the mean values from the posterior distributions, if a point (single) estimate is desired; however, the estimate to the full posterior may be used to place "error bars" on the inferred mean matrices $S$ and $\boldsymbol{L}$, of interest when making subsequent classification/tracking inferences, for example. An advantage of the Bayesian model is that prior knowledge can be flexibly incorporated, with minor changes to the model structure and computations. For example, 
the flexibility of the Bayesian framework allows us to exploit anticipated structure in the sparse component. As an example, consider video analysis, with the video frames defining the matrix columns. Assume we attempt to extract temporally and spatially localized moving objects (sparse component) from the static (or near-static) background (low-rank component), in the presence of frame-dependent additive noise $\boldsymbol{E}$. It is desirable to account for the correlation in the properties of the sparse part of the video, from frame to frame (column to column in the matrix). In this paper, we assume a Markov dependency in time and space for the sparse component, which implies a Markov property between the sparse components of consecutive matrix columns. Within a Bayesian framework, this structure is readily imposed, with the Markov properties inferred via the observed matrix (i.e., the form of the Markov model is imposed, with the data used to infer the Markov parameters).

In related statistical analysis of matrices, a probabilistic interpretation of PCA has been introduced, termed probabilistic PCA [19]. Based on this framework, Bayesian models for robust PCA have been proposed [20], [21]. Rather than considering the sparse outliers in $\boldsymbol{S}$ as discussed above, the work in [20], [21] considers outliers existing in the dense noise $\boldsymbol{E}$. Therefore these models assume $\boldsymbol{Y}=\boldsymbol{L}+\boldsymbol{E}$, with the noise term $\boldsymbol{E}$ modeled by heavy-tailed distributions, such as Laplacian distribution [20] or the Student-t distribution [21]. Although these types of distributions may describe outliers in dense noise, the models do not have the ability to distinguish sparse outliers from small dense noise, especially if the sparse outliers are of particular interest.

The work presented here is also closely related to low-rank matrix completion (for matrices with missing entries). There has been significant recent interest in this problem, addressed based on convex optimization [22], [23] or based on a hierarchical Bayesian framework [24], [25]. The objective of the problem is to approximate a matrix (with noisy entries) by a low-rank matrix and to predict the missing entries. In a Bayesian framework, the low-rank matrix $\boldsymbol{L} \in \mathbb{R}^{N \times M}$ can be decomposed into two matrices, i.e., $\boldsymbol{L}=\boldsymbol{U} \boldsymbol{V}$, with $\boldsymbol{U} \in \mathbb{R}^{N \times r}$ and $\boldsymbol{V} \in \mathbb{R}^{r \times M}$, where $r$ is the rank of $\boldsymbol{L}$. Note that this matrix factorization form is the SVD decomposition of $\boldsymbol{L}$ in (1) if the singular-value matrix $\Lambda$ is absorbed by $D$ and/or $\boldsymbol{W}$. Because of the flexibility of the Bayesian framework, many variants of this matrix factorization form have been proposed to improve the model robustness and performance [26], [27]. Although we do not consider this here, if the matrix under test $\boldsymbol{Y}=\boldsymbol{L}+\boldsymbol{S}+\boldsymbol{E}$ is missing entries at random, the proposed model can consider this as well, and can make estimates for the missing entries (in terms of the low- 
rank term $\boldsymbol{L}$ ). The $\boldsymbol{S}$ term may be viewed as defining a sparse set of matrix entries that should be removed when estimating $\boldsymbol{L}$; in the matrix completion problem the location of these entries - the missing entries - are of course known, while in the robust PCA problem the location of $\boldsymbol{S}$ must be inferred, while also estimating values of $\boldsymbol{L}, \boldsymbol{S}$ and $\boldsymbol{E}$.

This paper is also related to recent developments in Bayesian compressive sensing [28], [29], [30], which aims to achieve sparse signal reconstruction. Typically, in a Bayesian framework, the sparseness-promoting prior (e.g., a hierarchical normal-gamma prior in [28]) is imposed on the signal of interest, and the posterior distribution of the sparse signal is inferred. Known statistical structure within the sparse coefficients can be readily embedded in the model. For example, the work in [31], [32] has explicitly imposed a hidden Markov tree structure within the wavelet decomposition of an image. The manner in which we track $S$ from frame to frame in video is related to the tree structure used in [31].

The remainder of the paper is organized as follows. In Section II we present the proposed Bayesian low-rank and sparse model, and relate it to the optimization-based approaches in the literature. In Section III the Markov dependency for the sparse component is introduced, of particular interest in video applications. Section IV provides the computational inference methods considered for the model, with experimental results presented in Section V. We conclude in Section VI.

\section{BAYESIAN LOW-RANK AND SPARSE MODEL}

We assume that the observed data matrix $\boldsymbol{Y} \in \mathbb{R}^{N \times M}$ is the superposition of three parts: low-rank component $\boldsymbol{L} \in \mathbb{R}^{N \times M}$, sparse component $\boldsymbol{S} \in \mathbb{R}^{N \times M}$, and noise term $\boldsymbol{E} \in \mathbb{R}^{N \times M}$. The sparse component $\boldsymbol{S}$ may have large magnitude (relative to the magnitude of $\boldsymbol{L}$ ) but sparse support (only a small fraction of entries in $\boldsymbol{S}$ are nonzero), and $\boldsymbol{E}$ has generally small magnitude but dense support. Given observation $\boldsymbol{Y}=\boldsymbol{L}+\boldsymbol{S}+\boldsymbol{E}$, our objective is to recover $\boldsymbol{L}$ and $\boldsymbol{S}$.

The proposed Bayesian model is

$$
\boldsymbol{Y}=\boldsymbol{D}(\boldsymbol{Z} \boldsymbol{\Lambda}) \boldsymbol{W}+\boldsymbol{B} \circ \boldsymbol{X}+\boldsymbol{E}
$$

where $\boldsymbol{D} \in \mathbb{R}^{N \times K}, \boldsymbol{W} \in \mathbb{R}^{K \times M}, \boldsymbol{\Lambda} \in \mathbb{R}^{K \times K}$ is a diagonal matrix, $\boldsymbol{X} \in \mathbb{R}^{N \times M}$, and $\circ$ denotes the Hadamard (pointwise) product. The diagonal matrix $Z$ has binary entries along the diagonal, and the binary matrix $\boldsymbol{B} \in\{0,1\}^{N \times M}$ is sparse. The integer $K$ defines the largest possible rank that may be inferred for $\boldsymbol{L}$, and $K$ is set to a large value. Below we explain the model in detail. 


\section{A. Low-rank component}

The low-rank component is modeled as $\boldsymbol{L}=\boldsymbol{D}(\boldsymbol{Z} \boldsymbol{\Lambda}) \boldsymbol{W}$. Note that this is similar to the SVD decomposition in (1), but here we introduce an extra diagonal matrix $\boldsymbol{Z}$, with diagonal elements $z_{k k} \in\{0,1\}$ for $k=1, \ldots, K$. The product of the two matrices, $\boldsymbol{Z} \boldsymbol{\Lambda}$, is still a diagonal matrix and plays a role analogous to the singular values of $\boldsymbol{L}$. The introduction of $\boldsymbol{Z}$ decouples the rank learning and the singular-value learning, in that the magnitudes of the singular values are inferred in $\Lambda$ and the rank is learned as $r=\|\boldsymbol{Z}\|_{0}$. Since the rank of $\boldsymbol{L}$ is unknown, we choose large $K$, and hence we expect that the diagonal entries of $\boldsymbol{Z} \Lambda$ are sparse (if they are not, we know the selected $K$ was not large enough).

The binary diagonal matrix $Z$ is modeled as

$$
z_{k k} \sim \operatorname{Bernoulli}\left(p_{k}\right), \quad p_{k} \sim \operatorname{Beta}\left(\alpha_{0}, \beta_{0}\right), \quad k=1, \ldots K
$$

with hyperparameters $\alpha_{0}>0$ and $\beta_{0}>0$. The sparseness of the diagonal of $Z$ is explicitly imposed through hyperparameters $\alpha_{0}$ and $\beta_{0}$. Note that $\mathbb{E}\left[p_{k}\right]=\alpha_{0} /\left(\alpha_{0}+\beta_{0}\right)$, where $\mathbb{E}[\cdot]$ denotes the expectation of a random variable. To impose the sparseness on the diagonal of $Z$, we may let $\alpha_{0} /\left(\alpha_{0}+\beta_{0}\right) \ll 1$, and therefore it is probable that $p_{k}$ is close to zero. In our experiments we specify $\alpha_{0}=1 / K$ and $\beta_{0}=(K-1) / K$.

Each diagonal entry in $\Lambda$, denoted as $\lambda_{k k}$ for $k=1, \ldots, K$, is drawn from a normal-gamma distribution:

$$
\lambda_{k k} \sim \mathcal{N}\left(0, \tau^{-1}\right), \quad k=1, \ldots, K, \quad \tau \sim \operatorname{Gamma}\left(a_{0}, b_{0}\right)
$$

with hyperparameters $a_{0}>0$ and $b_{0}>0$. In (5) the we set $a_{0}=b_{0}=10^{-6}$ [33].

The columns of matrices $\boldsymbol{D}=\left[\boldsymbol{d}_{1}, \ldots, \boldsymbol{d}_{K}\right]$ and $\boldsymbol{W}=\left[\boldsymbol{w}_{1}, \ldots, \boldsymbol{w}_{M}\right]$ are assumed to be drawn from normal distributions:

$$
\begin{array}{rlrl}
\boldsymbol{d}_{k} & \sim \mathcal{N}\left(\mathbf{0}, \frac{1}{N} \boldsymbol{I}_{N}\right), & & k=1, \ldots, K \\
\boldsymbol{w}_{m} \sim \mathcal{N}\left(\mathbf{0}, \frac{1}{K} \boldsymbol{I}_{K}\right), & m & =1, \ldots, M
\end{array}
$$

where $\boldsymbol{I}_{N}$ denotes an $N \times N$ identity matrix. Note that when $N$ and $K$ are large the columns of $\boldsymbol{D}$ are approximately orthogonal to each other (also true for the columns of $\boldsymbol{W}$ ), consistent with their role in an SVD decomposition; however, here we only impose that the columns of $\boldsymbol{D}$ span the space of the principal vectors, without explicitly requiring orthonormality. One could 
explicitly impose orthonormality if desired [34], but this comes at significant computational cost and it has been found unnecessary in our experiments.

The decomposition of the low-rank component $\boldsymbol{L}=\boldsymbol{D}(\boldsymbol{Z} \boldsymbol{\Lambda}) \boldsymbol{W}$ can also be viewed from the standpoint of factor analysis. We may rewrite each column of $\boldsymbol{L}=\left[\boldsymbol{l}_{1}, \ldots, \boldsymbol{l}_{M}\right]$ as

$$
\boldsymbol{l}_{m}=\boldsymbol{D}(\boldsymbol{Z} \boldsymbol{\Lambda}) \boldsymbol{w}_{m}=\sum_{k=1}^{K} z_{k k} \lambda_{k k} w_{k m} \boldsymbol{d}_{k}, \quad m=1, \ldots, M .
$$

This suggests that each column of $\boldsymbol{L}$ can be viewed as a weighted sum of the dictionary elements (columns) in $\boldsymbol{D}$, and the $K$ can be seen as the size of the dictionary. The weights $\left\{z_{k k} \lambda_{k k}\right\}_{k=1: K}$ globally determine all the dictionary elements that are active to construct $\boldsymbol{L}$, and the weights $\left\{w_{k m}\right\}_{k=1: K}$ determine the importance of the selected dictionary elements for representation of the $m$ th column of $\boldsymbol{L}$. Equation (8) is also useful in the model inference, and will be revisited in Section IV.

\section{B. Sparse Component}

The sparse component is modeled as $\boldsymbol{S}=\boldsymbol{B} \circ \boldsymbol{X}$, where $\boldsymbol{B}$ is a binary matrix. Again, this approach separates the learning of sparseness from the learning of values, such that the zero component in $\boldsymbol{S}$ is exactly zero. Each column of the binary matrix $\boldsymbol{B}=\left[\boldsymbol{b}_{1}, \ldots, \boldsymbol{b}_{M}\right]$ is modeled as

$$
\boldsymbol{b}_{m} \sim \prod_{n=1}^{N} \operatorname{Bernoulli}\left(\pi_{n}\right), m=1, \ldots, M, \quad \pi_{n} \sim \operatorname{Beta}\left(\alpha_{1}, \beta_{1}\right), \quad n=1, \ldots N .
$$

Here the $n$th row in $\boldsymbol{B}$ shares a common prior parameter $\pi_{n}$. In addition, the model is robust to noise because updating $\pi_{n}$ uses all data in the $n$th row of $\boldsymbol{B}$. As an alternative approach, each entry in $\boldsymbol{B}$ may have its own prior parameter. This alternative is convenient for introducing further structure into the prior for the sparse component, such as a Markov property, as demonstrated in Section III. A sparseness prior is explicitly placed on $\boldsymbol{B}$, by specifying $\alpha_{1}$ and $\beta_{1}$ such that $\alpha_{1} /\left(\alpha_{1}+\beta_{1}\right) \ll 1$, as explained in (4). In our experiments we let $\alpha_{1}=1 / N$ and $\beta_{1}=(N-1) / N$.

The columns of $\boldsymbol{X}=\left[\boldsymbol{x}_{1}, \ldots, \boldsymbol{x}_{M}\right]$ are assumed drawn from a normal-gamma distribution:

$$
\boldsymbol{x}_{m} \sim \mathcal{N}\left(\mathbf{0}, \nu^{-1} \boldsymbol{I}_{N}\right), m=1, \ldots, M, \quad \nu \sim \operatorname{Gamma}\left(c_{0}, d_{0}\right)
$$

We let all the data in $\boldsymbol{X}$ share the same precision parameter $\nu$, to reduce model complexity and increase robustness. As in (5), typically we set $c_{0}=d_{0}=10^{-6}$. 


\section{Noise Component}

We assume the measurement noise is drawn i.i.d. from a Gaussian distribution, and the noise affects all the measurements. Note that this construction is only within the prior, and the approximation to the posterior of $\boldsymbol{E}$ can infer perturbations to this prior, if present, moving beyond the i.i.d. Gaussian model, if the data so indicates. The noise variance or precision is assumed unknown, and is learned within the model inference. Mathematically, the noise is modeled as

$$
e_{n m} \stackrel{i i d}{\sim} \mathcal{N}\left(0, \gamma^{-1}\right), n=1, \ldots, N, m=1, \ldots, M, \quad \gamma \sim \operatorname{Gamma}\left(e_{0}, f_{0}\right)
$$

where $e_{n m}$ denotes the entry at row $n$ and column $m$ of $\boldsymbol{E}$. As in (5) and (10), we set hyperparameters $e_{0}=f_{0}=10^{-6}$. One should note that while there appear to be many hyperparameters in the model, as indicated above they are all set in a "standard" manner [33], with no tuning performed or cross-validation required.

If desired, the model can learn different noise variances for different parts of $\boldsymbol{E}$. For example, in a video application, each column/row of $\boldsymbol{Y}$ corresponds to a video frame, and each frame may in general have its own noise level (we will consider this in the experiments). Let the data be organized such that each frame represents one column of $\boldsymbol{Y}$. We may then modify the noise structure in (11) as

$$
e_{n m} \sim \mathcal{N}\left(0, \gamma_{m}^{-1}\right), n=1, \ldots, N, \quad \gamma_{m} \sim \operatorname{Gamma}\left(e_{0}, f_{0}\right)
$$

for $m=1, \ldots, M$.

\section{Relation to optimization-based approach}

We now make connections of the Bayesian model proposed here to optimization-based approaches [10], [17], [18]. Note that for the low-rank component, we place a sparseness prior on the singular values, to impose a desire for a small number of non-zero singular values; in [17] the $\ell_{0}$ norm (number of non-zero elements) of the singular values is minimized in the original objective function, with this relaxed to an $\ell_{1}$ norm when solving the problem computationally. Similarly, for the sparse component, we place a sparseness prior to impose the belief that few components of $\boldsymbol{S}$ are nonzero, while in [17] the $\ell_{0}$ norm of the sparse component is introduced, with this again relaxed to an $\ell_{1}$ norm when performing computations. 
To see the connection more clearly, note that the negative logarithm of the full posterior density function of our Bayesian model is

$$
\begin{aligned}
-\log p(\boldsymbol{\Theta} \mid \boldsymbol{Y}, \mathcal{H}) & =\frac{\tau}{2}\|\boldsymbol{\Lambda}\|_{F}^{2}-\log \left[f_{B B}(\boldsymbol{Z} ; \mathcal{H})\right]+\frac{N}{2} \sum_{k=1}^{K}\left\|\boldsymbol{d}_{k}\right\|_{2}^{2}+\frac{1}{2} \sum_{m=1}^{M}\left\|\boldsymbol{w}_{m}\right\|_{2}^{2} \\
& +\frac{\nu}{2}\|\boldsymbol{X}\|_{F}^{2}-\log \left[f_{B B}(\boldsymbol{B} ; \mathcal{H})\right]+\frac{\gamma}{2}\|\boldsymbol{Y}-\boldsymbol{L}-\boldsymbol{S}\|_{F}^{2} \\
& -\log [\operatorname{Gamma}(\tau \mid \mathcal{H}) \operatorname{Gamma}(\nu \mid \mathcal{H}) \operatorname{Gamma}(\gamma \mid \mathcal{H})]+\text { Const. }
\end{aligned}
$$

where $\Theta$ represents all model parameters, $f_{B B}(\cdot \mid \mathcal{H})$ represents the beta-Bernoulli prior in (4) or (9), and $\mathcal{H}=\left\{\alpha_{0}, \beta_{0}, \alpha_{1}, \beta_{1}, a_{0}, b_{0}, c_{0}, d_{0}, e_{0}, f_{0}\right\}$ are model hyperparameters. Therefore, the error term $(2 \mu)^{-1}\|\boldsymbol{Y}-\boldsymbol{L}-\boldsymbol{S}\|_{F}^{2}$ in [18] (where the dense noise is considered) corresponds to the Gaussian prior placed on the measurement noise in (11). In [18] the authors suggest that the optimal balance parameter $\mu$ is a function of noise variance, which is consistent with the weight $\frac{\gamma}{2}$ here. However, in our Bayesian model, we are not required to know the noise variance a priori, and the model will learn the variance when performing inference. For the low-rank component, rather than employing an $\ell_{1}$ constraint to impose the sparseness of singular values, we employ a Gaussian prior to obtain a constraint on Frobenius matrix norm $\|\Lambda\|_{F}^{2}$, together with a beta-Bernoulli distribution to explicitly encourage the sparseness of singular values (like an $\ell_{0}$ prior). Similarly for the sparse component, instead of the $\ell_{1}$ constraint used in [10], [17], [18], the constraint on Frobenius matrix norm $\|\boldsymbol{X}\|_{F}^{2}$ and the beta-Bernoulli distribution are employed to impose the sparseness (again like an $\ell_{0}$ prior). An $\ell_{1}$ constraint may also be imposed in a Bayesian framework, by employing a Laplacian prior (implemented as in Bayesian Lasso [35]). However, the beta-Bernoulli prior employed here imposes exactly zero entries, while the $\ell_{1}$ Laplacian prior gives many small entries (but not exactly zero). Note also that the $\ell_{2}$ regularization in (13) for $\boldsymbol{d}_{k}$ and $\boldsymbol{w}_{m}$ has been used in previous related optimization approaches [36], and comes naturally as a consequence of the Gaussian priors on $\boldsymbol{d}_{k}$ and $\boldsymbol{w}_{m}$.

The differences notwithstanding, we note that there are strong connections between the Bayesian formulation and the optimization approaches in [10], [17], [18], [36]. Apart from the details in the differences in the form of $-\log p(\boldsymbol{\Theta} \mid \boldsymbol{Y}, \mathcal{H})$ relative to related optimization-based approaches, the main difference is that here we use numerical methods to estimate a distribution for the unknown parameters, while in optimization-based approaches one effectively seeks a single solution that minimizes a functional analogous to $-\log p(\boldsymbol{\Theta} \mid \boldsymbol{Y}, \mathcal{H})$ (ideally with convexity guarantees [10], 
[17], [18], [36]).

\section{Markov Dependency of Sparse Term in Time And Space}

In the prior, given parameters $\boldsymbol{\pi}=\left\{\pi_{n}\right\}_{n=1: N}$, the entries in the sparse component $\boldsymbol{S}$ are assumed to be drawn independently. This is clearly not true for some applications. For example, in fixed-camera video-surveillance analysis, the (nearly) static background manifests the low-rank component, and the moving objects are the sparse component. It is clear that the moving objects are strongly correlated across consecutive frames, and therefore the independence assumption is inappropriate.

We assume the appearance of the sparse component across frames (time) satisfies a Markov property. Specifically, if there is a moving object at some place in a frame, it is very likely that this object will appear at about the same place in the next frame. Let the sparse component of frame $t$ ( $t$ indexes time) be $\boldsymbol{F}_{t}$, with the pixel at coordinates $(i, j)$ denoted $F_{t}(i, j)$. The parent node of $F_{t}(i, j)$ is defined as $F_{t-1}(i, j)$, i.e., the sparse component at the same coordinates in the previous frame. We may then exploit the belief that if a parent node is non-zero, with a high probability its child node is also non-zero.

The simple Markov structure imposed above reflects the anticipated dependence imposed by a moving object across frames. However it is very sensitive to noise. If a noise entry exists in $\boldsymbol{F}_{t}$, it will, with high probability, affect all the later frames. To make the Markov dependency more robust to noise, we introduce a spatial dependence. We define the neighbor of $F_{t}(i, j)$ as $\mathbb{N}\left[F_{t}(i, j)\right]=\left\{F_{t}\left(i^{\prime}, j^{\prime}\right):\left|i^{\prime}-i\right| \leq R a,\left|j^{\prime}-j\right| \leq R a\right\}$, and in our experiments we set $R a=1$. Other definitions for a spatial neighborhood may also be imposed. We then define the state of $F_{t}(i, j)$, denoted as $\mathbb{S}\left[F_{t}(i, j)\right]$ :

$$
\mathbb{S}\left[F_{t}(i, j)\right]= \begin{cases}\text { active, } & \text { if }\left\|\mathbb{N}\left[F_{t}(i, j)\right]\right\|_{0} \geq \rho \\ \text { inactive, } & \text { otherwise }\end{cases}
$$

where $\rho$ is a predefined scalar; we set $\rho=5$ in our experiments. This definition imposes that we consider a node to be in an active state if the sparse component contains at least $\rho$ nonzero members in its neighborhood. The Markov dependency between a parent and its child is now modified as: if a parent node is active, then with a high probability its child node is also active. In this way the sparsity of a child node depends not only on its parent node (in time) but also on the neighbors of its parent node (in space). 
With the temporal and spatial assumptions discussed above, we modify the basic model to impose the dependency on the sparse component $\boldsymbol{S}$. Assume the $t$ th column of $\boldsymbol{Y}$ is constructed by concatenating all components of frame $t$, for $1 \leq t \leq M$. The Markov dependency is then imposed by modifying (9) as

$$
\begin{aligned}
\boldsymbol{b}_{t} & \sim \prod_{n=1}^{N} \operatorname{Bernoulli}\left(\pi_{n t}\right), t=1, \ldots, M, \\
\pi_{n t} & \sim\left\{\begin{array}{ll}
\operatorname{Beta}\left(\alpha_{H}, \beta_{H}\right) & \text { if } \mathbb{S}\left[b_{n, t-1}\right]=\text { active }, \\
\operatorname{Beta}\left(\alpha_{L}, \beta_{L}\right) & \text { if } \mathbb{S}\left[b_{n, t-1}\right]=\text { inactive, }
\end{array} \quad n=1, \ldots N, t=2, \ldots, M .\right.
\end{aligned}
$$

For $t=1$, we may still use (9) since there are no parent nodes for the first frame. The subscripts $H$ and $L$ in the model above recall the "high" and "low" states in the Markov tree model used for wavelet coefficients in compressive sensing [31]. To determine $\mathbb{S}\left[b_{n, t-1}\right]$, the coordinates $(i, j)$ can be calculated from $n$. We let $\alpha_{H} /\left(\alpha_{H}+\beta_{H}\right) \approx 1$ and $\alpha_{L} /\left(\alpha_{L}+\beta_{L}\right) \approx 0$, so that the sparseness pattern propagates along time with high probability. Note that a component of $\boldsymbol{S}$ is not directly non-zero depending on whether it is active/inactive; rather, if a component is in an active state it has a high probability of being non-zero, while if it is in an inactive state it has a low probability of being non-zero.

\section{POSTERIOR INFERENCE}

In the proposed hierarchical model, the density function at one layer is conjugate to the density function at the layer above it. This greatly eases the ability to perform computations. Specifically, we perform computations for an approximation to the posterior density function in two ways: via Markov chain Monte Carlo (MCMC) analysis [37] implemented using a Gibbs sampler, and via the variational Bayesian (VB) approximation [38]. In MCMC the posterior distribution is approximated by a set of samples, collected by iteratively drawing each random variable (model parameters) from its conditional posterior distribution given the most recent values of all the other parameters. The MCMC-based Bayesian inference for the model (3)-(11) is summarized in Algorithm 1, where $\boldsymbol{y}_{m}$ represents the $m$ th column in $\boldsymbol{Y}$ and $(a \mid-)$ represents random variable $a$ given all the other random variables in the model. The algorithm starts from a set of initial values $\Theta_{0}$, and after a large number of burn-in iterations $N_{\text {burn-in }}$, we consider the Markov chain

has become stable. A number $N_{\text {collect }}$ of samples $\left\{\boldsymbol{\Theta}^{(i)}\right\}_{i=1: N_{\text {collect }}}$ are then collected to represent the joint posterior distribution of these random variables. 
Algorithm 1: MCMC-based Bayesian Inference.

$\left\{\boldsymbol{\Theta}^{(i)}\right\}_{i=1: N_{\text {collect }}}=\operatorname{BRPCAmcmc}\left(\boldsymbol{Y}, \boldsymbol{\Theta}_{0}, \mathcal{H}, N_{\text {burn-in }}, N_{\text {collect }}\right)$

$\Theta \leftarrow \Theta_{0}$

For iter $=1$ to $N_{\text {burn-in }}+N_{\text {collect }}$

$\%$ low-rank component

For $k=1$ to $K$

$$
\begin{aligned}
& \tilde{\boldsymbol{y}}_{m}^{-k} \leftarrow \boldsymbol{y}_{m}-\boldsymbol{D}(\boldsymbol{Z} \boldsymbol{\Lambda}) \boldsymbol{w}_{m}-\boldsymbol{b}_{m} \circ \boldsymbol{x}_{m}+z_{k k} \lambda_{k k} w_{k m} \boldsymbol{d}_{k}, \text { for } m=1, \ldots, M \\
& \left(\boldsymbol{d}_{k} \mid-\right) \sim \mathcal{N}(\boldsymbol{\mu}, \boldsymbol{\Sigma}), \boldsymbol{\Sigma}=\left(N \boldsymbol{I}_{N}+\gamma \sum_{m=1}^{M} \lambda_{k k}^{2} z_{k k}^{2} w_{k m}^{2}\right)^{-1}, \boldsymbol{\mu} \sim \gamma \boldsymbol{\Sigma} \sum_{m=1}^{M}\left(\lambda_{k k} z_{k k} w_{k m} \tilde{\boldsymbol{y}}_{m}^{-k}\right) \\
& \left(z_{k k} \mid-\right) \sim \operatorname{Bernoulli}\left(\frac{q_{1}}{q_{0}+q_{1}}\right), q_{1}=p_{k} \exp \left(-\frac{\gamma}{2} \sum_{m=1}^{M}\left(\lambda_{k k}^{2} w_{k m}^{2} \boldsymbol{d}_{k}^{T} \boldsymbol{d}_{k}-2 \lambda_{k k} w_{k m} \boldsymbol{d}_{k}^{T} \tilde{\boldsymbol{y}}_{m}^{-k}\right)\right), q_{0}=1-p_{k} \\
& \left(\lambda_{k k} \mid-\right) \sim \mathcal{N}(\mu, \Sigma), \Sigma=\left(\tau+\gamma \sum_{m=1}^{M} z_{k k}^{2} w_{k m}^{2} \boldsymbol{d}_{k}^{T} \boldsymbol{d}_{k}\right)^{-1}, \mu=\gamma \Sigma \sum_{m=1}^{M} z_{k k} w_{k m} \boldsymbol{d}_{k}^{T} \tilde{\boldsymbol{y}}_{m}^{-k} \\
& \left(w_{k m} \mid-\right) \sim \mathcal{N}(\mu, \Sigma), \Sigma=\left(1+\gamma \lambda_{k k}^{2} z_{k k}^{2} \boldsymbol{d}_{k}^{T} \boldsymbol{d}_{k}\right)^{-1}, \mu=\gamma \Sigma \lambda_{k k} z_{k k} \boldsymbol{d}_{k}^{T} \tilde{\boldsymbol{y}}_{m}^{-k}, \text { for } m=1, \ldots, M \\
& \left(p_{k} \mid-\right) \sim \operatorname{Beta}\left(\alpha_{0}+z_{k k}, \beta_{0}+1-z_{k k}\right)
\end{aligned}
$$

\section{End}

$(\tau \mid-) \sim \operatorname{Gamma}\left(a_{0}+0.5 K, b_{0}+0.5 \sum_{k=1}^{K} \lambda_{k k}^{2}\right)$

$\%$ sparse component

$\tilde{\boldsymbol{y}}_{m}^{s} \leftarrow \boldsymbol{y}_{m}-\boldsymbol{D}(\boldsymbol{Z} \boldsymbol{\Lambda}) \boldsymbol{w}_{m}$, for $m=1, \ldots, M$

$\left(b_{n m} \mid-\right) \sim \operatorname{Bernoulli}\left(\frac{q_{1}}{q_{0}+q_{1}}\right), q_{1}=\pi_{n} \exp \left(-\frac{\gamma}{2} x_{n m}^{2}-2 x_{n m} \tilde{y}_{n m}^{s}\right), q_{0}=1-\pi_{n}$, for $n=1, \ldots, N$, $m=1, \ldots, M$

$\left(x_{n m} \mid-\right) \sim \mathcal{N}(\mu, \Sigma), \Sigma=\left(\nu+\gamma b_{n m}^{2}\right)^{-1}, \mu=\gamma \Sigma b_{n m} \tilde{y}_{n m}^{s}$, for $n=1, \ldots, N, m=1, \ldots, M$

$\left(\pi_{n} \mid-\right) \sim \operatorname{Beta}\left(\alpha_{1}+\sum_{m=1}^{M} b_{n m}, \beta_{1}+M-\sum_{m=1}^{M} b_{n m}\right)$, for $n=1, \ldots, N$

$(\nu \mid-) \sim \operatorname{Gamma}\left(c_{0}+0.5 N M, d_{0}+0.5 \sum_{m=1}^{M} \boldsymbol{x}_{m}^{T} \boldsymbol{x}_{m}\right)$

$\%$ noise component

$(\gamma \mid-) \sim \operatorname{Gamma}\left(e_{0}+0.5 N M, f_{0}+0.5\|\boldsymbol{Y}-\boldsymbol{D}(\boldsymbol{Z} \boldsymbol{\Lambda}) \boldsymbol{W}-\boldsymbol{B} \circ \boldsymbol{X}\|_{F}^{2}\right)$

$\%$ collecting samples

$$
\text { If } \text { iter }>N_{\text {burn-in }}, \boldsymbol{\Theta}^{\left(i t e r-N_{\text {burn-in }}\right)} \leftarrow \boldsymbol{\Theta}, \text { End }
$$

\section{End}

In Algorithm 1, the random variables $D, Z, \Lambda$ and $\boldsymbol{W}$ in the low-rank component are updated with $K$ iterations, by considering the contribution from only the $k$ th dictionary element at iteration $k$, as explained in (8). In this approach, the learning of matrices is decoupled into the learning of correlated vectors. This is not employed when we update the random variables 
of the sparse component, because the entries in the sparse component are assumed independent. Furthermore, we factorize $p\left(\boldsymbol{w}_{m}\right)$ in the inference, $p\left(\boldsymbol{w}_{m}\right)=\prod_{k=1}^{K} p\left(w_{k m}\right)$, to avoid the inversion of a large matrix, reducing the computational burden. Note that with the MCMC inference, this factorization of $p\left(\boldsymbol{w}_{m}\right)$ does not mean that the entries of $\boldsymbol{W}$ are independent, since each random variable is drawn from a distribution conditioned on all other random variables.

For the model with Markov dependency imposed on the sparse component, Algorithm 1 is only slightly modified when sampling $b_{n m}$ and $\pi_{n m}$, for $n=1, \ldots, N, m=1, \ldots, M$. Specifically, we have:

$$
\begin{aligned}
\left(b_{n m} \mid-\right) \sim \operatorname{Bernoulli}\left(\frac{q_{1}}{q_{0}+q_{1}}\right), q_{1}=\pi_{n m} \exp \left(-\frac{\gamma}{2} x_{n m}^{2}-2 x_{n m} \tilde{y}_{n m}^{s}\right), q_{0}=1-\pi_{n m}, \\
\left(\pi_{n m} \mid-\right) \sim \begin{cases}\operatorname{Beta}\left(\alpha_{1}+b_{n m}, \beta_{1}+1-b_{n m}\right) & \text { if } m=1 \\
\operatorname{Beta}\left(\alpha_{H}+b_{n m}, \beta_{H}+1-b_{n m}\right) & \text { if } m>1, \mathbb{S}\left[b_{n, m-1}\right]=\text { active }, \\
\operatorname{Beta}\left(\alpha_{L}+b_{n m}, \beta_{L}+1-b_{n m}\right) & \text { if } m>1, \mathbb{S}\left[b_{n, m-1}\right]=\text { inactive. }\end{cases}
\end{aligned}
$$

The variational Bayesian (VB) inference employs a set of distributions $q(\boldsymbol{\Theta})$ to approximate the true posterior distributions $p(\boldsymbol{\Theta} \mid \boldsymbol{Y})$, and uses a lower bound $\mathcal{F}$ to approximate the true $\log$ likelihood of the model $\log p(\boldsymbol{Y} \mid \boldsymbol{\Theta})$. The algorithm iteratively updates $q(\boldsymbol{\Theta})$ so that $\mathcal{F}$ approaches to $\log p(\boldsymbol{Y} \mid \boldsymbol{\Theta})$ until convergence. Here we omit the update equations for simplicity. Readers may refer to [38] for details on VB inference.

For the proposed model the computational complexity of a single MCMC and VB iteration is approximately the same. However, the MCMC method requires a large number of burn-in iterations, followed by a sufficient number of iterations to collect $\Theta$ samples. By contrast, the VB method directly updates the hyperparameters of $q(\boldsymbol{\Theta})$, and therefore the latest update of $q(\boldsymbol{\Theta})$ represents the distribution of $\Theta$. After tens of iterations the VB algorithm usually converges, with convergence inferred by monitoring the quantitative criterion - lower bound $\mathcal{F}$. However, as mentioned above, the VB solution may find a local-optimal solution (it is not guaranteed to converge to a globally-optimal best solution). We have found Gibbs sampling to work quite effectively in practice for this problem, with the mean of the desired parameters converging relatively quickly (even when the number of samples is too small to accurately represent the full posterior density function). Therefore, all results presented below will be for the Gibbs sampler, with discussions also provided that summarize our experience with the VB analysis. 


\section{EXPERIMENTAL RESUlts}

\section{A. Simulation}

Reconstruction accuracy. We first demonstrate the performance of the proposed algorithm on simulated data. The data are generated similar to that in [17]. We consider square matrices of varying dimension $N=500,1000,1500$. The low-rank component $\boldsymbol{L} \in \mathbb{R}^{N \times N}$ is generated as a product of two independent $N \times r$ matrices whose elements are drawn i.i.d. from $\mathcal{N}(0,1 / N)$. The sparse component $\boldsymbol{S} \in \mathbb{R}^{N \times N}$ is generated such that the non-zero elements are located uniformly at random and the amplitudes are drawn uniformly at random in the range $[-1,1]$. The observation $\boldsymbol{Y}=\boldsymbol{L}+\boldsymbol{S}+\boldsymbol{E}$, with elements of $\boldsymbol{E}$ drawn i.i.d. from $\mathcal{N}\left(0, \sigma^{2}\right)$. We consider two situations: a noise free case with $\sigma=0$ and a noisy case with $\sigma=10^{-4}$.

The proposed model (3)-(11) is applied to infer the distributions of the low-rank and sparse components, with estimates denoted $\hat{\boldsymbol{L}}$ and $\hat{\boldsymbol{S}}$, respectively. We set the largest possible rank as $K=200$, and the model hyperparameters are specified as follows: $a_{0}=b_{0}=c_{0}=d_{0}=e_{0}=$ $f_{0}=10^{-6}, \alpha_{0}=1 / K, \beta_{0}=(K-1) / K, \alpha_{1}=0.1 N$ and $\beta_{1}=0.9 N$. The reconstruction accuracy are shown in Tables I(a) and II(a), for the noise free case and the noisy case, respectively. All results are based on MCMC inference with 25000 burn-in iterations followed by 5000 collection samples. In Tables I(a) and II(a), we show results for the mean values of estimated $\hat{\boldsymbol{L}}$ and $\hat{\boldsymbol{S}}$, but we have access to "error bars", which will be demonstrated later in a real video example.

In Tables I and II three metrics are used to evaluate the reconstruction accuracy of the algorithm: $\operatorname{rank}(\hat{\boldsymbol{L}}),\|\hat{\boldsymbol{S}}\|_{0}$ and $\frac{\|\hat{\boldsymbol{L}}-\boldsymbol{L}\|_{F}}{\|\boldsymbol{L}\|_{F}}$. A good reconstruction should have accurate estimate of $\operatorname{rank}(\hat{\boldsymbol{L}})$ and $\|\hat{\boldsymbol{S}}\|_{0}$, and at the same time have small reconstruction errors for the low-rank component, evaluated as $\frac{\|\hat{\boldsymbol{L}}-\boldsymbol{L}\|_{F}}{\|\boldsymbol{L}\|_{F}}$. The reconstruction error for the sparse component, $\frac{\|\hat{\boldsymbol{S}}-\boldsymbol{S}\|_{F}}{\|\boldsymbol{S}\|_{F}}$, is usually small as long as the number of nonzero elements in the sparse component is estimated accurately; therefore it is not considered here. From Tables I(a) and II(a) we observe that the proposed algorithm is able to successfully estimate $\boldsymbol{L}$ and $\boldsymbol{S}$ with either noise-free or noisy observation, even with $10 \%$ of the elements in the sparse components nonzero.

As a comparison, in Tables I(b) and II(b)-(c) we show results on the same data set from a state-of-the-art algorithm: optimization-based robust PCA algorithm proposed in [10] ${ }^{1}$. Recall

\footnotetext{
${ }^{1} \mathrm{We}$ used the MATLAB codes of exact_alm_rpca package [39], downloaded from http://watt.csl.illinois.edu/ perceive/matrix-rank/home.html .
} 
(a) Bayesian robust PCA

\begin{tabular}{|c|c|c|c|c|c|}
\hline$N$ & $\operatorname{rank}(\boldsymbol{L})$ & $\|\boldsymbol{S}\|_{0}$ & $\operatorname{rank}(\hat{\boldsymbol{L}})$ & $\frac{\|\hat{\boldsymbol{L}}-\boldsymbol{L}\|_{F}}{\|\boldsymbol{L}\|_{F}}$ & $\|\hat{\boldsymbol{S}}\|_{0}$ \\
\hline 500 & 25 & 12500 & 25 & $1.1 \times 10^{-4}$ & 12499 \\
1000 & 50 & 50000 & 50 & $9.5 \times 10^{-5}$ & 49998 \\
1500 & 75 & 112500 & 75 & $1.2 \times 10^{-4}$ & 112492 \\
\hline 500 & 25 & 25000 & 25 & $1.2 \times 10^{-4}$ & 24999 \\
1000 & 50 & 100000 & 50 & $9.9 \times 10^{-5}$ & 99996 \\
1500 & 75 & 225000 & 75 & $1.1 \times 10^{-4}$ & 224985 \\
\hline
\end{tabular}

(b) Optimization-based robust PCA, Tol $=10^{-6}$

\begin{tabular}{|c|c|c|c|c|c|}
\hline$N$ & $\operatorname{rank}(\boldsymbol{L})$ & $\|\boldsymbol{S}\|_{0}$ & $\operatorname{rank}(\hat{\boldsymbol{L}})$ & $\frac{\|\hat{\boldsymbol{L}}-\boldsymbol{L}\|_{F}}{\|\boldsymbol{L}\|_{F}}$ & $\|\hat{\boldsymbol{S}}\|_{0}$ \\
\hline 500 & 25 & 12500 & 25 & $3.6 \times 10^{-6}$ & 12505 \\
1000 & 50 & 50000 & 50 & $2.0 \times 10^{-6}$ & 50000 \\
1500 & 75 & 112500 & 75 & $3.8 \times 10^{-6}$ & 112496 \\
\hline 500 & 25 & 25000 & 25 & $3.5 \times 10^{-6}$ & 25002 \\
1000 & 50 & 100000 & 50 & $4.3 \times 10^{-6}$ & 100013 \\
1500 & 75 & 225000 & 75 & $5.4 \times 10^{-6}$ & 225008 \\
\hline
\end{tabular}

TABLE I

COMPARISON OF RECONSTRUCTION ACCURACY FOR NOISE-FREE OBSERVATION $(\sigma=0)$. THE TRUE RANK OF THE MATRIX

$\boldsymbol{L}$ IS SET AS $5 \% N$, AND THE NUMBER OF NONZERO SPARSE ELEMENTS IS $5 \% N^{2}$ AND $10 \% N^{2}$ FOR THE TOP AND THE BOTTOM OF EACH TABLE, RESPECTIVELY.

that in [10] the model is assumed $\boldsymbol{Y}=\boldsymbol{L}+\boldsymbol{S}$ and there is no noise term. However, the algorithm requires a pre-specified tolerance Tol as a stop criterion. When $\frac{\|\boldsymbol{Y}-\hat{\boldsymbol{L}}-\hat{\boldsymbol{S}}\|_{F}}{\|\boldsymbol{Y}\|_{F}}<T o l$, the algorithm stops. This tolerance is clearly related to the noise level the model assumes when solving for $\boldsymbol{L}$ and $\boldsymbol{S}$. In the noise-free situation, we set a small tolerance $T o l=10^{-6}$, and the reconstruction is very accurate (see Table I(b)). However, we observe that in a noisy situation, the optimization-based algorithm is very sensitive to this tolerance setting. In Table II(b), when Tol $=7.3 \times 10^{-4}$, the model gives a good reconstruction, but if we change the tolerance from $7.3 \times 10^{-4}$ to $7.2 \times 10^{-4}$, the estimation of the rank and the number of sparse elements fails badly (see Table II(c)). By contrast, the proposed Bayesian model is robust to the noise, with no tuning or optimization required for the model hyperparameters (the same hyperparameters are used for all examples, both noisy and noise-free, toy and real).

It should be pointed out that on this toy data set the computation time for the proposed model is much longer than that from the optimization-based algorithm. This is because the 
(a) Bayesian robust PCA

\begin{tabular}{|c|c|c|c|c|c|}
\hline$N$ & $\operatorname{rank}(\boldsymbol{L})$ & $\|\boldsymbol{S}\|_{0}$ & $\operatorname{rank}(\hat{\boldsymbol{L}})$ & $\frac{\|\hat{\boldsymbol{L}}-\boldsymbol{L}\|_{F}}{\|\boldsymbol{L}\|_{F}}$ & $\|\hat{\boldsymbol{S}}\|_{0}$ \\
\hline 500 & 25 & 12500 & 25 & $4.5 \times 10^{-3}$ & 12495 \\
1000 & 50 & 50000 & 50 & $6.4 \times 10^{-3}$ & 49990 \\
1500 & 75 & 112500 & 75 & $7.9 \times 10^{-3}$ & 112500 \\
\hline 500 & 25 & 25000 & 25 & $4.7 \times 10^{-3}$ & 24994 \\
1000 & 50 & 100000 & 50 & $6.6 \times 10^{-3}$ & 100002 \\
1500 & 75 & 225000 & 75 & $8.1 \times 10^{-3}$ & 225011 \\
\hline
\end{tabular}

(b) Optimization-based robust PCA, Tol $=7.3 \times 10^{-4}$

\begin{tabular}{|c|c|c|c|c|c|}
\hline$N$ & $\operatorname{rank}(\boldsymbol{L})$ & $\|\boldsymbol{S}\|_{0}$ & $\operatorname{rank}(\hat{\boldsymbol{L}})$ & $\frac{\|\hat{\boldsymbol{L}}-\boldsymbol{L}\|_{F}}{\|\boldsymbol{L}\|_{F}}$ & $\|\hat{\boldsymbol{S}}\|_{0}$ \\
\hline 500 & 25 & 12500 & 25 & $3.4 \times 10^{-3}$ & 12501 \\
1000 & 50 & 50000 & 50 & $5.1 \times 10^{-3}$ & 49951 \\
1500 & 75 & 112500 & 75 & $6.5 \times 10^{-3}$ & 112380 \\
\hline
\end{tabular}

(c) Optimization-based robust PCA, Tol $=7.2 \times 10^{-4}$

\begin{tabular}{|c|c|c|c|c|c|}
\hline$N$ & $\operatorname{rank}(\boldsymbol{L})$ & $\|\boldsymbol{S}\|_{0}$ & $\operatorname{rank}(\hat{\boldsymbol{L}})$ & $\frac{\|\hat{\boldsymbol{L}}-\boldsymbol{L}\|_{F}}{\|\boldsymbol{L}\|_{F}}$ & $\|\hat{\boldsymbol{S}}\|_{0}$ \\
\hline 500 & 25 & 12500 & 58 & $3.4 \times 10^{-2}$ & 42474 \\
1000 & 50 & 50000 & 119 & $4.9 \times 10^{-3}$ & 169985 \\
1500 & 75 & 112500 & 172 & $5.9 \times 10^{-3}$ & 377216 \\
\hline
\end{tabular}

TABLE II

RECONSTRUCTION ACCURACY FOR NOISY OBSERVATION, WITH NOISE STANDARD DEVIATION $\sigma=10^{-4}$. FOR SIMPLICITY THE RESULTS FOR $\|\boldsymbol{S}\|_{0}=10 \% N^{2}$ ARE NOT SHOWN IN (B) AND (C).

computational complexity of the model is $\mathcal{O}(K(N+M)+M N)$, and if $K$ is large this is expensive (we specified $K=200$ ). In addition, the MCMC convergence speed is closely related to the true rank of $\boldsymbol{L}$. A large rank results in a slow convergence. However, in a real application, for example, video surveillance, usually the rank of $\boldsymbol{L}$ is very low. We may therefore select a relatively small $K$, and the MCMC convergence is fast. In this situation the computational time is comparable to that from the optimization-based algorithm; this is demonstrated below.

Robustness to noise. To further examine the robustness of the proposed model to noisy observations, we fix $N=100, \operatorname{rank}(\boldsymbol{L})=5,\|\boldsymbol{S}\|_{0}=500$, let the elements of two independent $N \times r$ matrices whose product generates $\boldsymbol{L}$ are drawn i.i.d. from $\mathcal{N}(0,1)$, and let the magnitudes of the non-zero elements in $\boldsymbol{S}$ are drawn uniformly in the range $[-500,500]$. We let the noise standard deviation change from $\sigma=0$ to $\sigma=1$ with step size 0.01 . Figure 1 plots the reconstruction quality as a function of noise level, solved with the proposed model and using 
the optimization-based model in [10]. For the optimization-based algorithm, since the results are sensitive to the tolerance, we set three different values for the tolerance: $T o l=0.001,0.01$ and 0.03 .

In Figure 1 the reconstruction quality is represented by $\operatorname{rank}(\hat{\boldsymbol{L}}),\|\hat{\boldsymbol{S}}\|_{0}$ and $\frac{\|\hat{\boldsymbol{L}}-\boldsymbol{L}\|_{F}}{\|\boldsymbol{L}\|_{F}}$. For the proposed model, we also plot the inferred noise standard deviation $\hat{\sigma}$ (mean value). It is observed from Figure 1(a) that the Bayesian model may adaptively estimate the noise variance, and gives good reconstruction results. We notice that the Bayesian rank estimate is not perfect occasionally. By examining the result, we find that when the inferred $\operatorname{rank}(\hat{\boldsymbol{L}})=6$, the sixth singular value is very small. Therefore, it has minimal impact to the reconstruction quality, which can be verified from $\|\hat{\boldsymbol{S}}\|_{0}$ and $\frac{\|\hat{\boldsymbol{L}}-\boldsymbol{L}\|_{F}}{\|\boldsymbol{L}\|_{F}}$. As a comparison, given a fixed tolerance, the optimizationbased algorithm [10] produces a good reconstruction only in a certain region of noise variance (see Figure 1(b) and (c)). In Figure 1(d) although the estimated $\operatorname{rank}(\hat{\boldsymbol{L}})$ and $\|\hat{\boldsymbol{S}}\|_{0}$ are accurate, the reconstruction error $\frac{\|\hat{\boldsymbol{L}}-\boldsymbol{L}\|_{F}}{\|\boldsymbol{L}\|_{F}}$ is always large, even for small noise variance. In general, for the optimization-based algorithm [10] if the noise variance is unknown, the tolerance has to be tuned carefully to obtain a good result (which may require human interpretation of the results to define an appropriate setting, since in practice "truth" is unknown).

Phase transition in rank, sparsity and noise level. We next investigate the algorithm's ability to recover matrices $\boldsymbol{L}$ and $\boldsymbol{S}$ for varying ranks, sparsity and noise levels. We consider square matrices of dimension $N=200$. The underlying matrices $\boldsymbol{L}$ and $\boldsymbol{S}$ are generated using the same procedure as used to generate simulated data for Tables I and II, with the rank and the sparsity defined according to $\rho_{r}=\operatorname{rank}(\boldsymbol{L}) / N$ and $\rho_{s}=\|\boldsymbol{S}\|_{0} / N^{2}$, respectively. We let $\rho_{r}$ vary from 0.05 to 0.45 with step size 0.05 , let $\rho_{s}$ vary with the same range and step size, and the noise standard deviation $\sigma_{n}$ varies in $\left[10^{-5}, 10^{-4}, 10^{-3}, 10^{-2}\right]$. For each $\left(\rho_{r}, \rho_{s}, \sigma_{n}\right)$ tuple, ten random problems are generated, each of which is solved via the proposed Bayesian robust PCA. We consider matrices $\boldsymbol{L}$ and $\boldsymbol{S}$ being successfully recovered if the recovered $\hat{\boldsymbol{L}}$ satisfies $\frac{\|\boldsymbol{L}-\hat{\boldsymbol{L}}\|_{F}}{\|\boldsymbol{L}\|_{F}} \leq T h$, where $T h$ is a threshold depending on the noise level. In our experiments, when the noise is very weak, e.g., $\sigma_{n}=10^{-5}$, the threshold $T h$ is set as $10^{-3}$ (which is the same as that in [17] for the noise free case); for noise standard deviations $\sigma_{n}=10^{-4}, 10^{-3}$ and $10^{-2}$, we select $T h=5 \times 10^{-3}, 5 \times 10^{-2}$, and $5 \times 10^{-1}$, respectively.

Figure 2 shows the fraction of successful recoveries for each $\left(\rho_{r}, \rho_{s}\right)$ pair, computed by Bayesian robust PCA, and for noise levels $\sigma_{n}=10^{-5}, 10^{-4}, 10^{-3}$ and $10^{-2}$. As a comparison, 


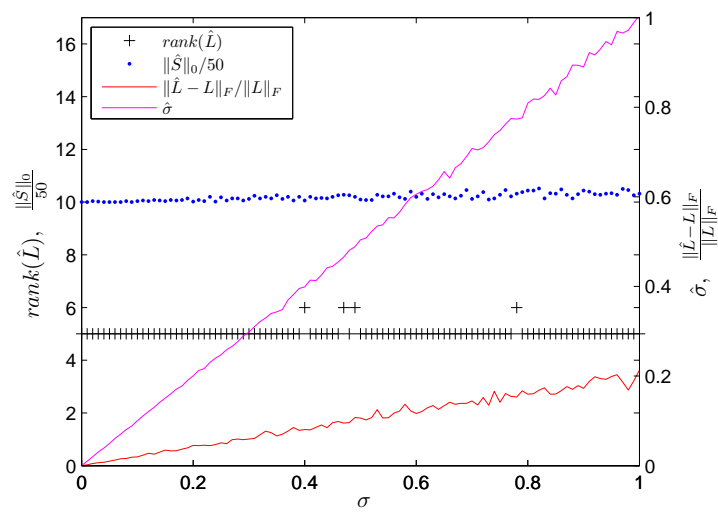

(a) Bayesian robust PCA

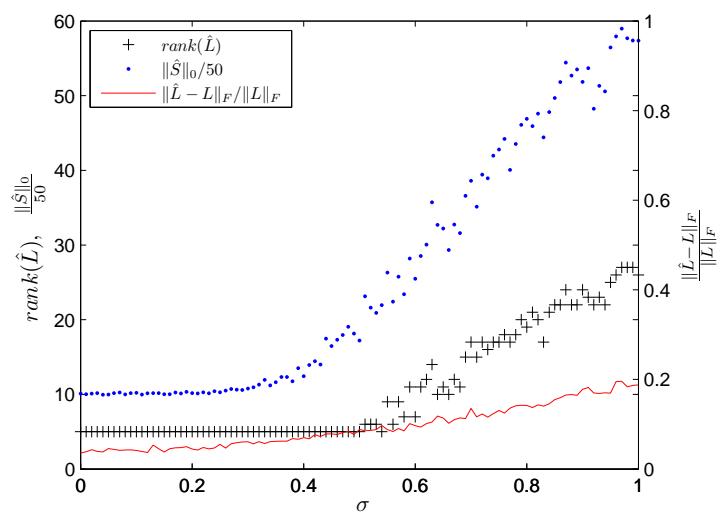

(c) Optimization-based robust PCA, Tol $=0.01$

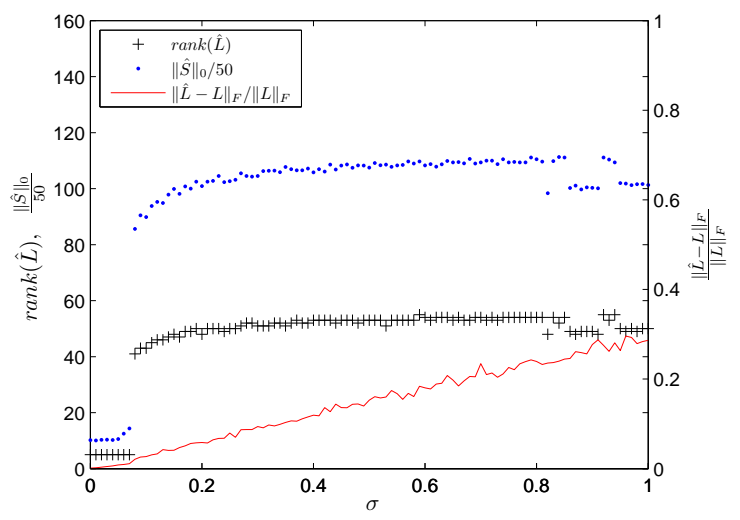

(b) Optimization-based robust PCA, Tol $=0.001$

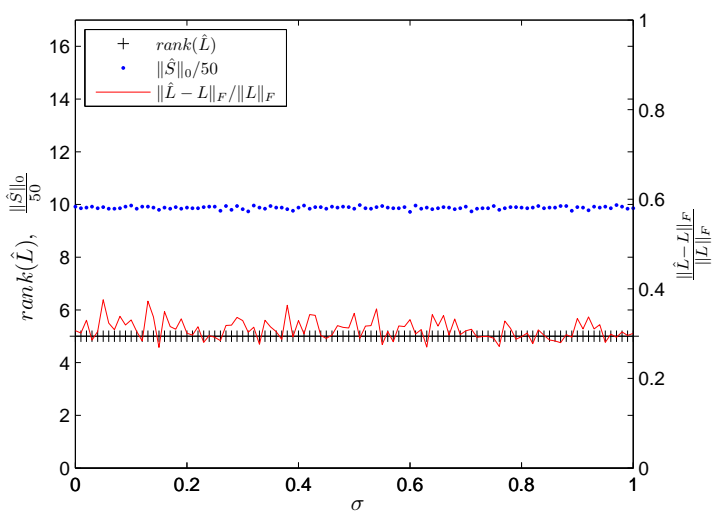

(d) Optimization-based robust PCA, Tol $=0.03$

Fig. 1. Reconstruction quality with respect to noise level under noisy environment. The left $y$-axis indicates $\operatorname{rank}(\hat{\boldsymbol{L}})$ and $\frac{\|\hat{\boldsymbol{S}}\|_{0}}{50}$, and the right $y$-axis indicates $\frac{\|\hat{\boldsymbol{L}}-\boldsymbol{L}\|_{F}}{\|\boldsymbol{L}\|_{F}}$ and the estimated noise standard deviation $\hat{\sigma}$. For (b), (c) and (d), no $\hat{\sigma}$ values are estimated.

Figure 3 plots the results for $\sigma_{n}=10^{-5}$ and $10^{-3}$, computed by the optimization-based robust PCA, in which the stop criterion $T o l$ is carefully selected for each $\left(\rho_{r}, \rho_{s}, \sigma_{n}\right)$ tuple to achieve the best recovery quality. For simplicity the results for $\sigma_{n}=10^{-4}$ and $10^{-2}$ are not shown. From these figures, we observe that the Bayesian robust PCA outperforms the optimizationbased robust PCA, in that it may achieve successful recoveries with larger regions in the $\left(\rho_{r}, \rho_{s}\right)$ space for various noise levels. Furthermore, no tuning of the stop criteria is required. 


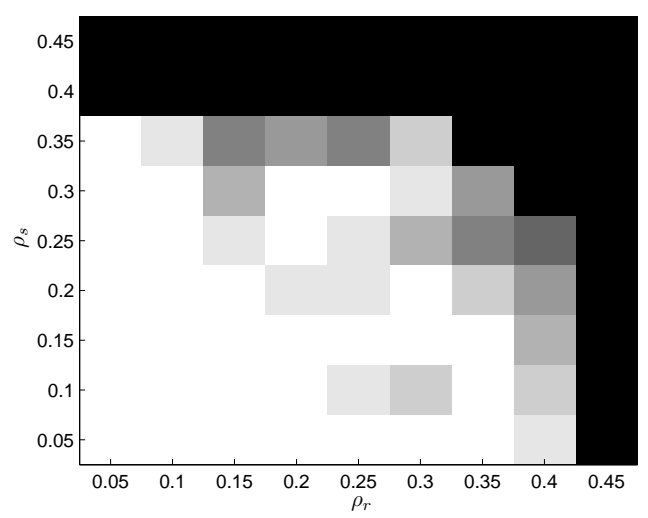

(a) $\sigma_{n}=10^{-5}$

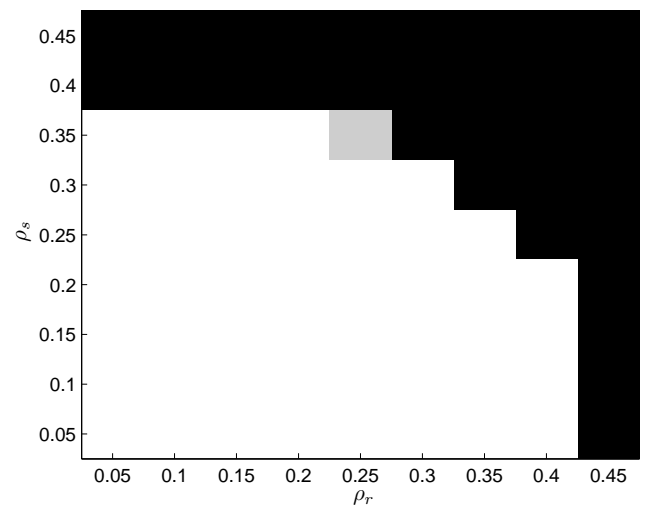

(c) $\sigma_{n}=10^{-3}$

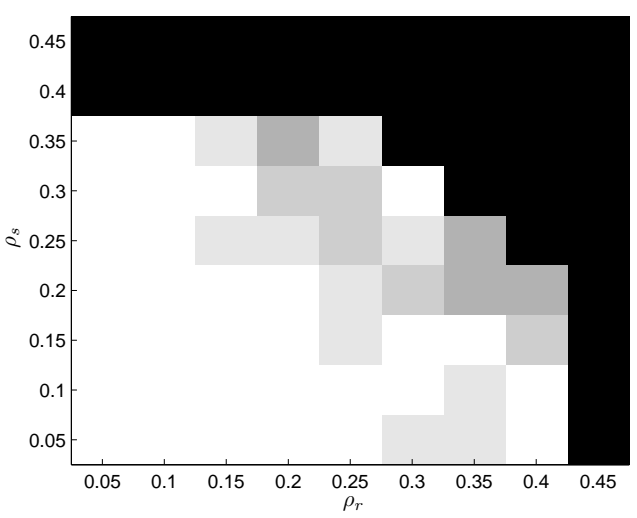

(b) $\sigma_{n}=10^{-4}$

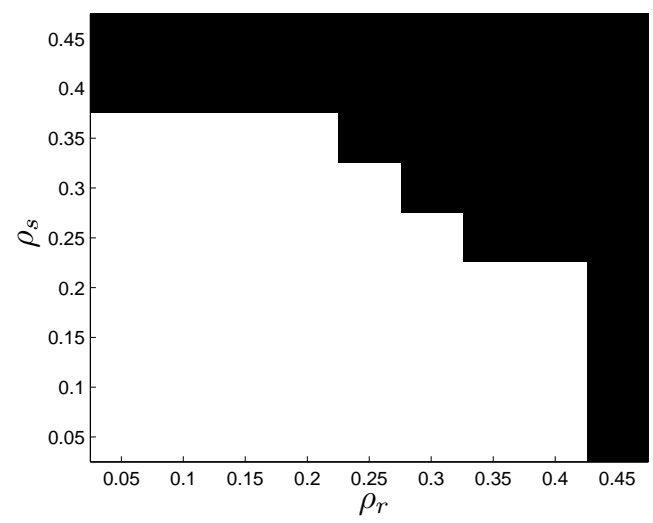

(d) $\sigma_{n}=10^{-2}$

Fig. 2. Fraction of successful recovery for varying ranks, sparsity, and noise levels, computed by Bayesian robust PCA. Given a tuple $\left(\rho_{r}, \rho_{s}, \sigma_{n}\right)$, white color represents that all the 10 trials are successfully recovered, and black means no trials is successfully recovered.

\section{B. Video example}

In this section we investigate the application of video surveillance with a fixed camera. The objective is to reconstruct a (near) static background and moving foreground from a video sequence. The data are organized such that column $m$ of $\boldsymbol{Y}$ is constructed by concatenating all pixels of frame $m$ from a grayscale video sequence. The background is then modeled as the lowrank component, and the moving foreground is modeled as the sparse component. As discussed above, the rank $r$ is usually small for a static background, and the sparse components across 


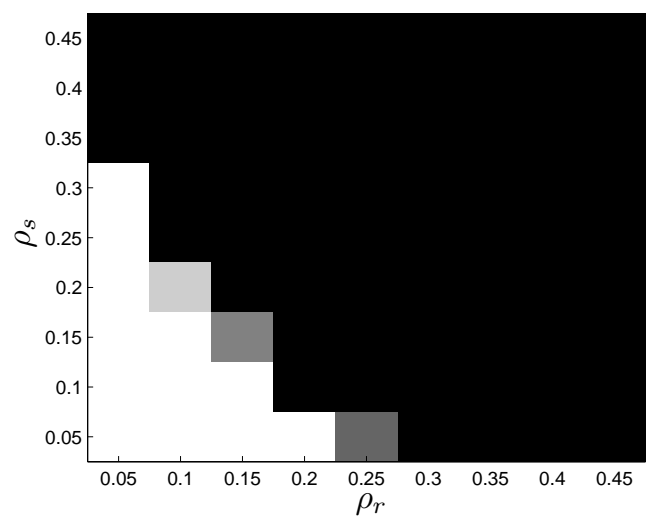

(a) $\sigma_{n}=10^{-5}$

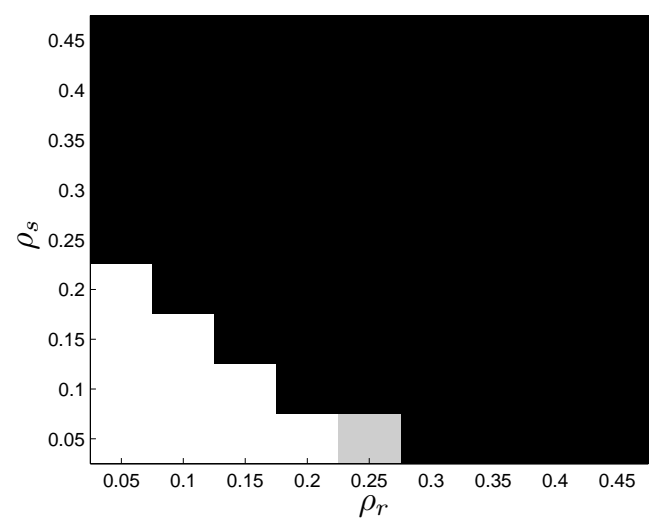

(b) $\sigma_{n}=10^{-3}$

Fig. 3. Fraction of successful recovery for varying ranks, sparsity, and noise levels, computed by optimization-based robust PCA.

frames (columns of $\boldsymbol{Y}$ ) are strongly correlated, modeled by the Markov dependency proposed in Section III. For all the video examples considered in this section, we set $K=20$, and the hyperparameters are specified the same as used in the simulation examples.

Reconstruction quality and time. In this experiment we consider a video sequence taken at the front door of a shop in a shopping center ${ }^{2}$. The video sequence consists of 118 frames of size $144 \times 192$ pixels. Figure 4 shows the reconstruction of the background $\boldsymbol{L}$ and the foreground $S$ for (arbitrarily selected) Frame 97, from the proposed basic model (3)-(11) and from the modified model with the Markov dependency imposed in (15). For comparison, we also show the reconstruction result from the optimization-based algorithm [10]. We find that when the observation is relatively noise-free, all the models produce good reconstruction results.

Next we assume the observation is noisy, with additive Gaussian white noise of standard deviation $\sigma=10$ (the image pixels have values in the range of $[0,255]$ ). Figure 5 shows the reconstruction from all three models. It is observed that when the noise is present, the modified model with Markov dependency obtains better results than the basic model (note that there are some isolated noisy points in the sparse component of the basic model). We also note that the optimization-based algorithm is sensitive to the parameter settings. If the tolerance is too small,

\footnotetext{
${ }^{2}$ The video sequence was downloaded at http://homepages.inf.ed.ac.uk/rbf/CAVIARDATA1/.
} 

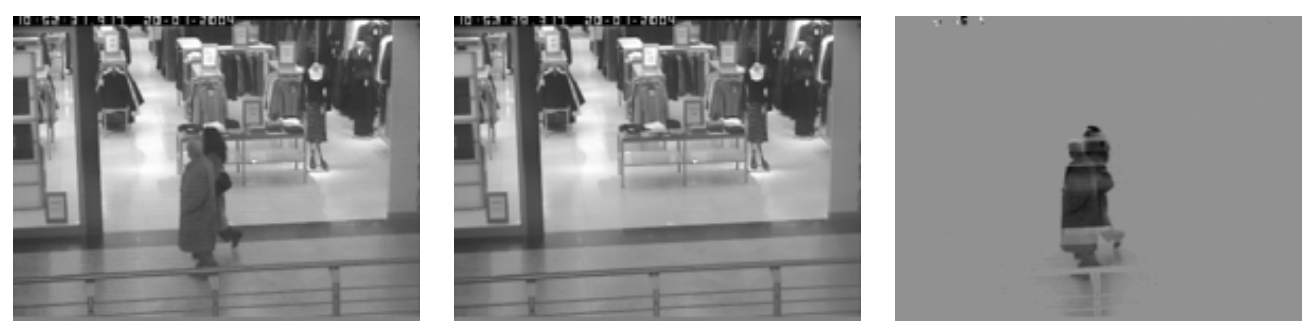

(a) Bayesian robust PCA with Markov dependency
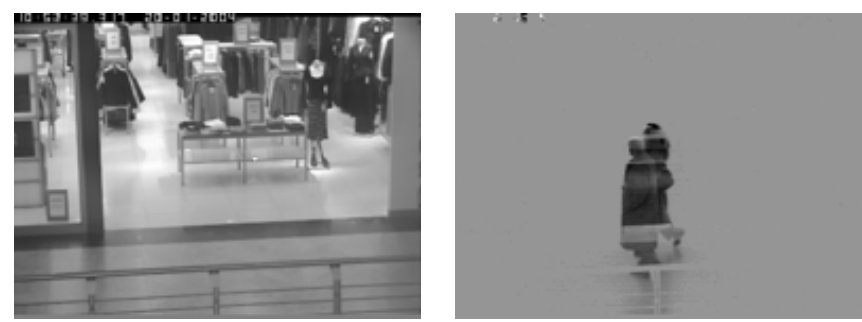

(b) Bayesian robust PCA, basic model
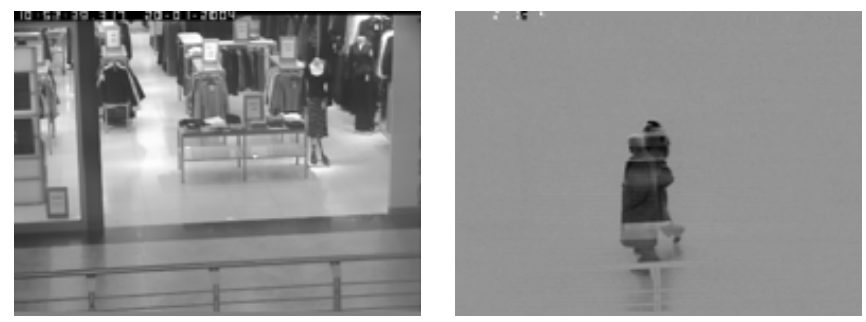

(c) Optimization-based robust PCA, Tol $=10^{-7}$

Fig. 4. Reconstruction of the background and the foreground. The video sequence contains 118 frames of size $144 \times 192$ pixels, and the results for Frame 97 are shown. Left column: original image; middle: reconstruction of the low-rank component (background); right: reconstruction of the sparse component (foreground).

the observation noise is counted in the sparse component (Figure 5(c)); when the tolerance is too large, the sparse component is absorbed by the observation noise (i.e., $\frac{\|\boldsymbol{Y}-\hat{\boldsymbol{L}}-\hat{\boldsymbol{S}}\|_{F}}{\|\boldsymbol{Y}\|_{F}}<$ Tol has already been satisfied even though $\hat{\boldsymbol{S}}=0$ ) and is therefore missing (Figure 5(e)). In this example a good reconstruction can be achieved only if the tolerance is carefully selected such that $0.06<$ Tol $<0.10$.

As mentioned above, the Bayesian model infers full posterior distributions of the model parameters. The plots in Figures 4 and 5 are the mean inferred $\hat{\boldsymbol{L}}$ and $\hat{\boldsymbol{S}}$. As an example, in Figure 6 we plot the error bars of the inferred $\hat{\boldsymbol{L}}$ and $\hat{\boldsymbol{S}}$, for the noisy observation case (the mean values are plotted in Figure 5(a)). The error bars represent one standard deviation of the posterior distribution for each pixel value in the two matrices. We observe that the background has uniformly tight error bars across the entire frame, while the moving object has relatively 

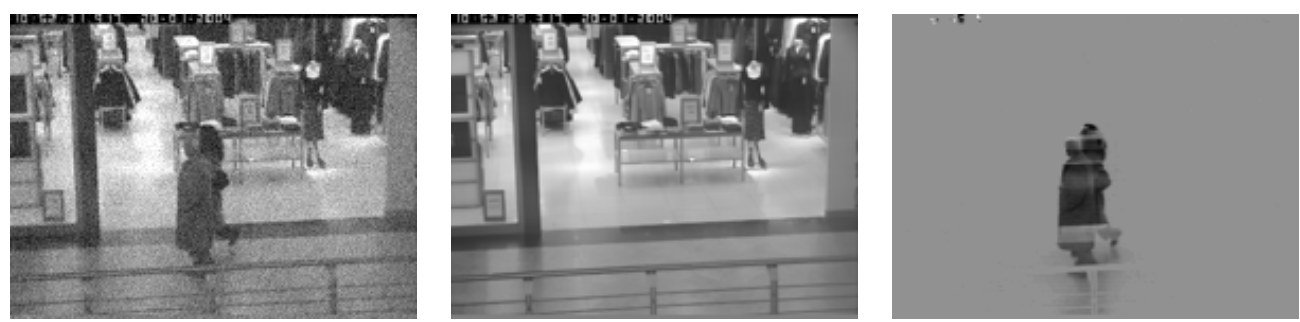

(a) Bayesian robust PCA with Markov dependency
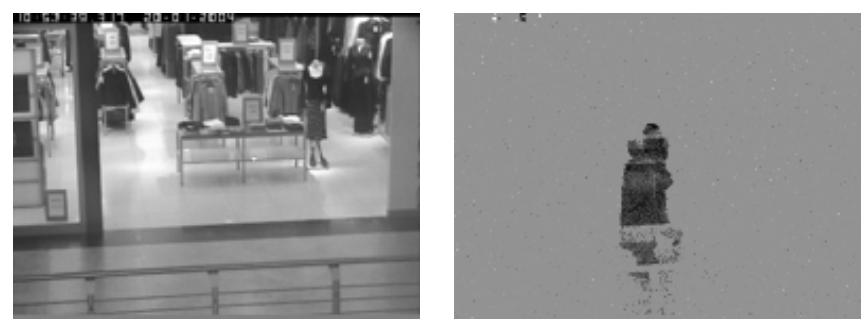

(b) Bayesian robust PCA, basic model
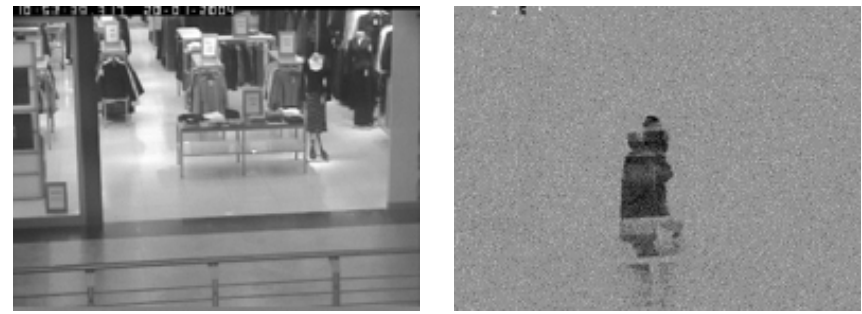

(c) Optimization-based robust PCA, Tol $=0.06$
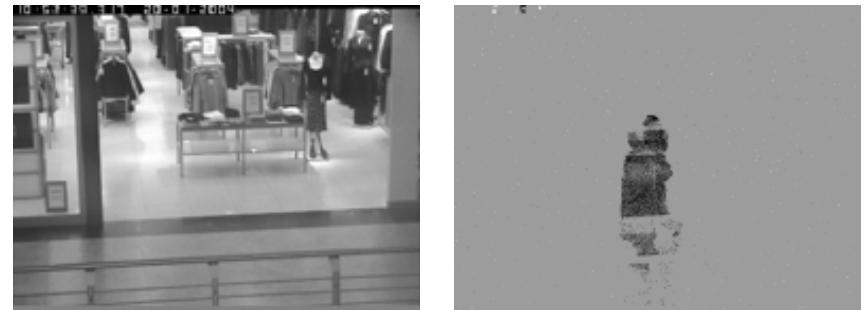

(d) Optimization-based robust PCA, Tol $=0.07$
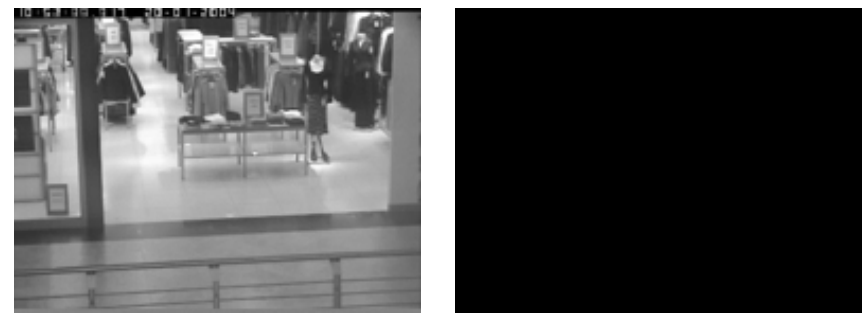

(e) Optimization-based robust PCA, Tol $=0.10$

Fig. 5. Reconstruction of the background and the foreground under noisy observation. The additive white Gaussian noise has standard deviation $\sigma=10$. Left: original noisy image; middle: background reconstruction; right: foreground reconstruction.

large error bars, since it has more uncertainty. 

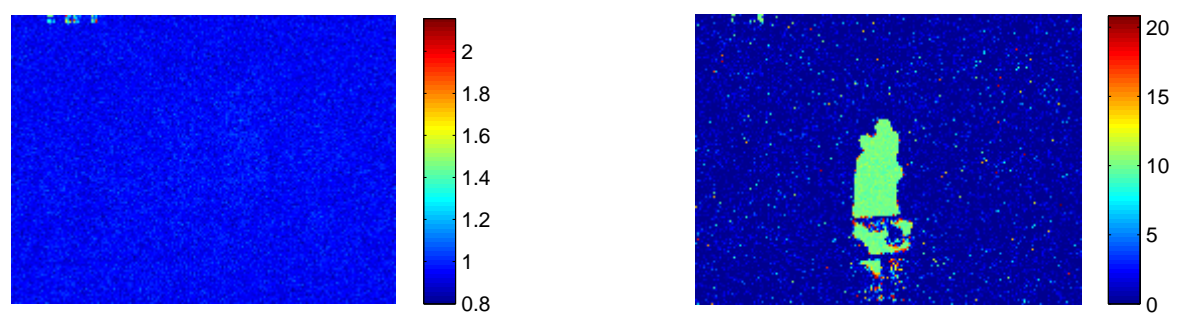

Fig. 6. Error bars associated with the results in Figure 5(a). The error bars represent one standard deviation of the posterior distribution for each inferred pixel value. Left: background; right: foreground.

In a video surveillance application, the rank is usually low, so that a small $K$ is appropriate (again, here we set $K=20$ ). We observe that the MCMC convergence is very fast, typically around 10 iterations. Therefore the computational speed of the proposed model is comparable to the optimization-based algorithm. Specifically, in the experiment performed in Figure 4, we let $N_{\text {burn-in }}=100$ and $N_{\text {collect }}=100$, and the computation time of the proposed model is 22 minutes. As a comparison, using the default tolerance $T o l=10^{-7}$ the optimization-based algorithm takes 19 minutes to produce the associated results.

Slow-changing foreground. If a foreground object moves slowly, it is more difficult to separate it from the static background. The model may treat the slow object as a part of the background, by increasing the rank of the low-rank component. In Figure 7 we demonstrate that the Bayesian model is relatively effective at distinguishing the slow foreground from the static background. We consider a video sequence consisting of 151 frames, which is a scene of a corridor in a shopping center ${ }^{3}$. Figure 7 shows the reconstruction of Frame 100 by the proposed algorithms and by the optimization-based algorithm. The two persons facing the camera are moving very slowly. We see that the optimization-based algorithm fails to completely separate the two persons from the background, while the proposed model, especially with Markov dependency imposed, more accurately recognizes them as foreground. This is because by imposing a sparseness prior on $Z$ the proposed model prefers a lower-rank background; in addition, the Markov property on the sparse component provides the model with more confidence on the slow changes of the foreground.

\footnotetext{
${ }^{3}$ The video sequence was downloaded at http://homepages.inf.ed.ac.uk/rbf/CAVIARDATA1/.
} 

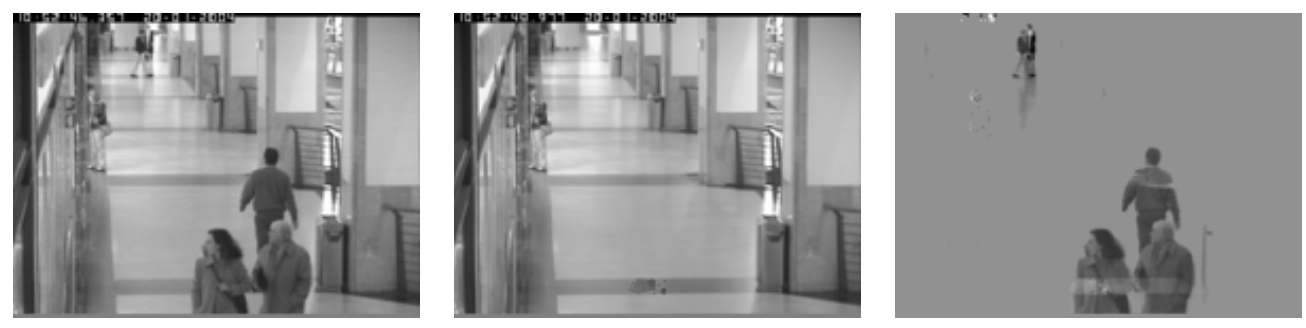

(a) Bayesian robust PCA with Markov dependency
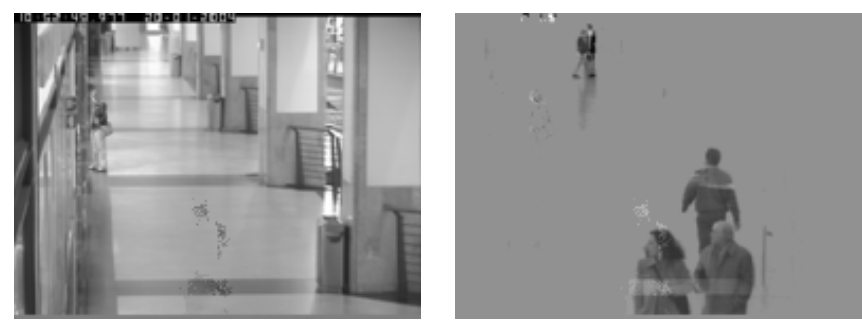

(b) Bayesian robust PCA, basic model
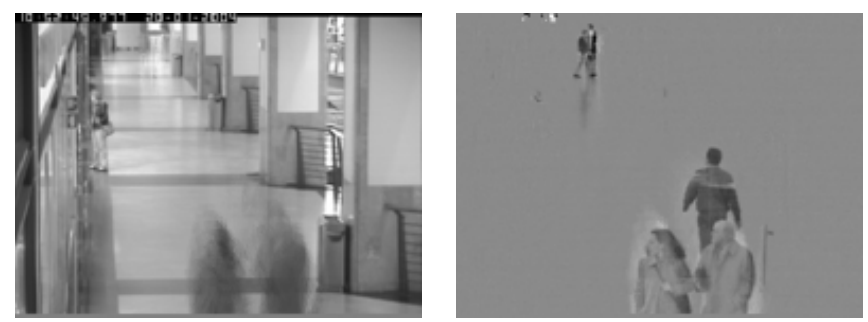

(c) Optimization-based robust PCA, Tol $=10^{-7}$

Fig. 7. Slow-changing foreground. The video contains 151 frames of size $144 \times 192$ pixels, and we show Frame 100 . The two persons closest to camera move very slowly. Left: original image; middle: background reconstruction; right: foreground reconstruction.

Non-stationary noise. Assume the noise variance varies across frames. We demonstrate that the Bayesian model can adaptively learn the changes of the noise variance during the inference. The model is modified as described in (12) for the noise term, and the noise precision $\gamma_{m}$, for $m=1, . ., M$, are inferred, each associated with one frame. We assume the noise standard deviation $\sigma_{m}$ is uniformly distributed in a range of [5, 15], i.e., $\sigma_{m} \stackrel{i . i . d}{\sim} U[5,15], m=1, \ldots, M$, and the noise precision $\gamma_{m}=1 / \sigma_{m}^{2}$. The Bayesian model with Markov dependency is applied. In Figure 8 we show the reconstruction of three frames: with smallest, median and largest noise variance, respectively. Figure 9 plots the true $\sigma_{m}$ and the inferred $\hat{\sigma}_{m}$. We observe that the proposed algorithm consistently obtains better estimate of noise variances across frames, without any parameter tuning. 

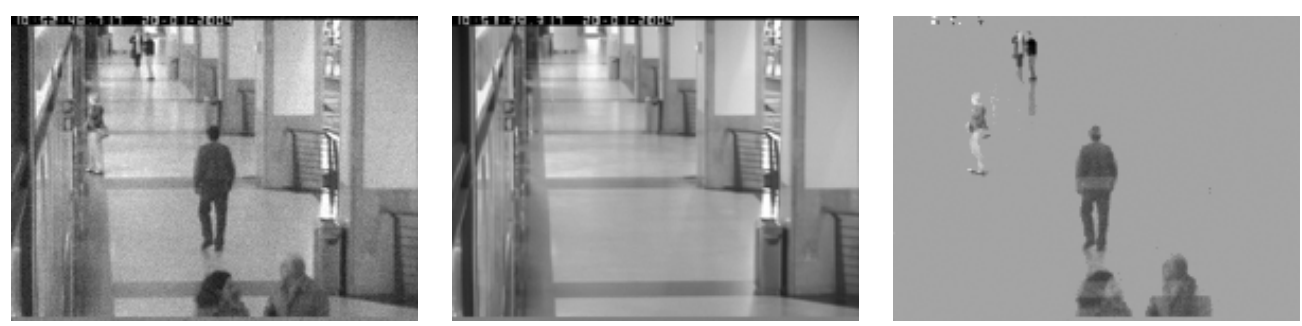

(a) Frame 152, smallest noise variance, $\sigma=5.0$
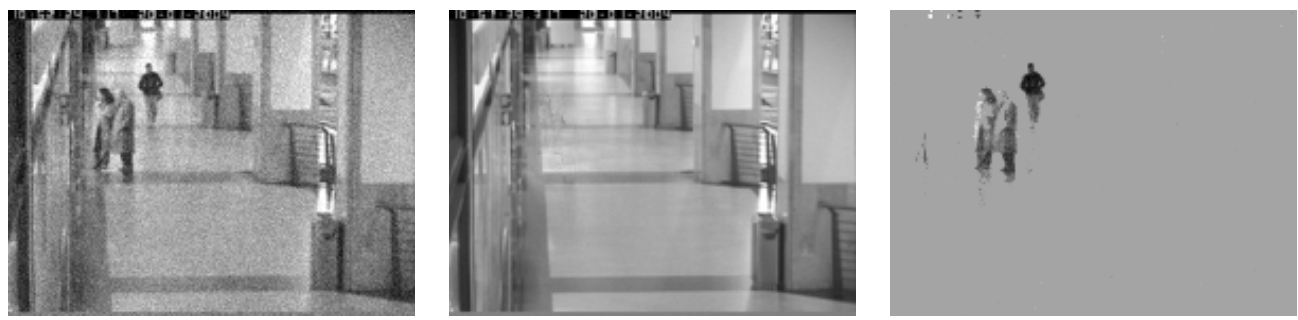

(b) Frame 111, median noise variance, $\sigma=9.9$
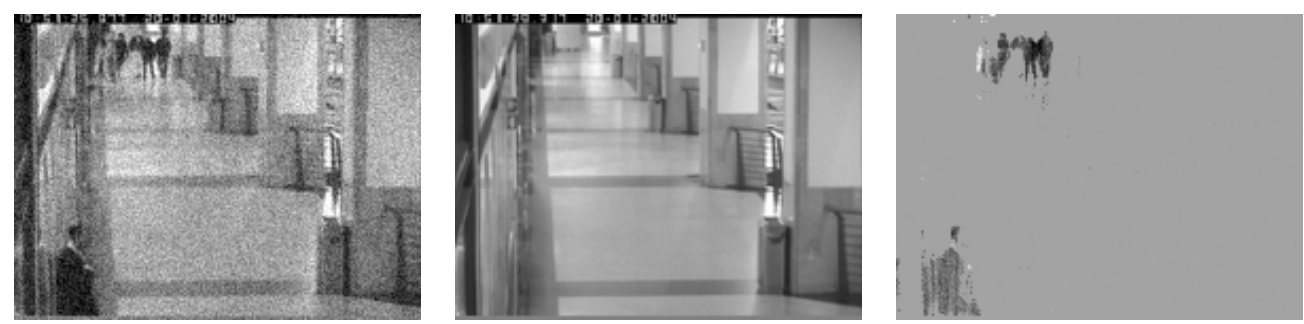

(c) Frame 14, largest noise variance, $\sigma=15.0$

Fig. 8. Non-stationary observation noise. The standard deviation of the noise is uniformly distributed in [5,15]. The reconstruction results are based on the Bayesian robust PCA with Markov dependency. Left: original image; middle: background reconstruction; right: foreground reconstruction.

Traffic video. Finally we examine another example: traffic viewed from a fixed video camera ${ }^{4}$. The video was taken at a street intersection; there are 300 frames, each of size $144 \times 192$ pixels. This is a more challenging data set because there are many activities (cars, buses, people, etc.), so that the sparse component is less sparse. In addition, during the time slot that the red traffic light is on, the cars in one direction stop. These stopped cars may be considered as a part of background. When the light turns green, these cars start moving, and they become a part of foreground. In Figure 10 we show the results from the Bayesian model and also from the optimization-based algorithm. By examining the detail in these figures, it can be observed that the proposed model outputs a clear separation of the background and the foreground, while

\footnotetext{
${ }^{4}$ The video sequence was downloaded at http://www.ngsim.fhwa.dot.gov/.
} 


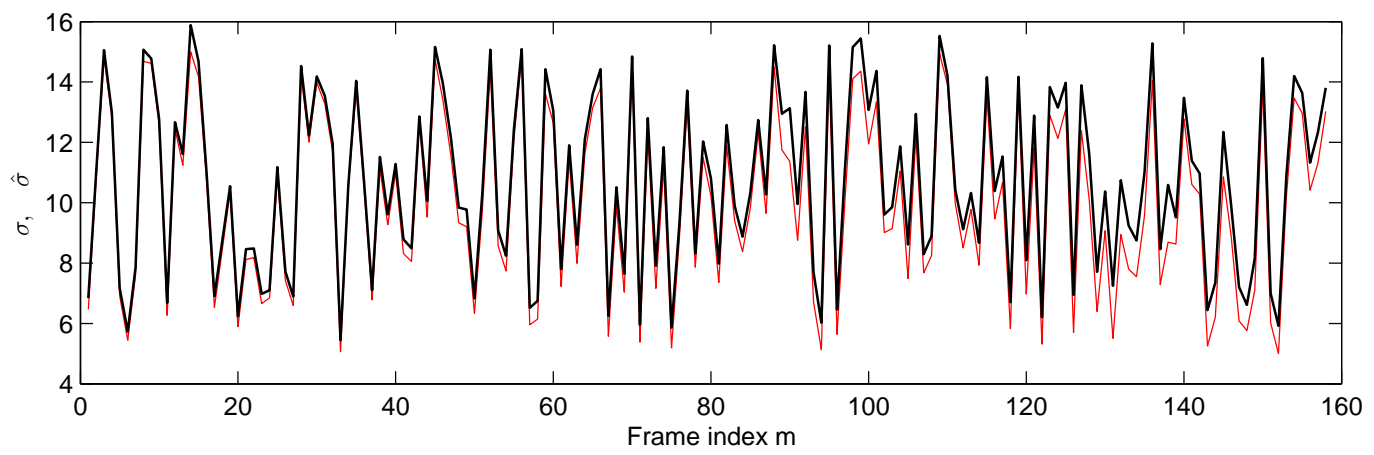

Fig. 9. True noise standard deviation $\sigma_{m}$ (red curve) and the inferred $\hat{\sigma}_{m}$ (black curve) in a non-stationary noisy environment.

the optimization-based algorithm is often ambiguous on the stopped cars (see the the vertical direction of Frame 212 (the last row of Figure 10), i.e., the stopped cars are present both in the background and in the foreground.
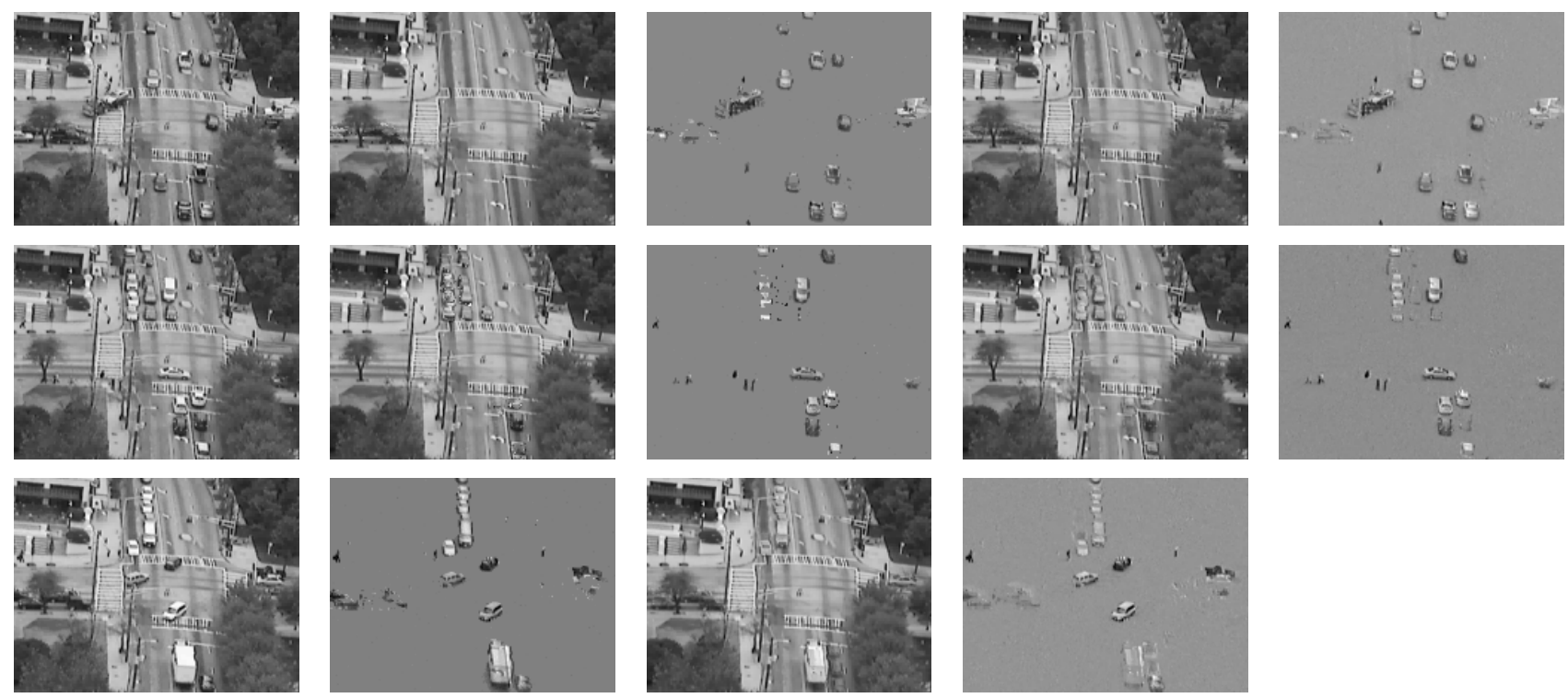

Fig. 10. Background and foreground reconstruction for traffic video. The video sequence consists of 300 frames of size $144 \times 192$ pixels. Three frames are shown. From top row to bottom row: Frame 10, Frame 110 and Frame 212 . From left column to right column: original image, background from Bayesian model, foreground from Bayesian model, background from optimization-based model, foreground from optimization-based model. 


\section{Discussion}

We also implemented the proposed Bayesian robust PCA model with variational Bayesian (VB) inference, as discussed in Section IV. However, we found that the VB inference for the model is often sensitive to model initialization (i.e., sensitive to local-optimal solutions). For the toy example, we have to specify the initial values very carefully, sometimes even with a "trialand-error method", to obtain the result comparable to that from the MCMC method, which requires no tuning and may be initialized with randomly selected parameter values. In the real examples, although the local-optimal-solution problem associated with VB is not as obvious as that in the toy examples, it still exists. In addition, one important advantage of the VB method fast convergence - becomes less attractive in the real examples, because the MCMC method also converges fast when the rank is low. Therefore, in practice it is felt that the Gibbs-sampler-based implementation is the most useful for the proposed model.

\section{CONClusions}

In [10], [17], [11] the authors developed a new robust PCA framework for analysis of matrices with sparely distributed additive noise of arbitrary amplitude, and in [18] the model was extended to consider small additive densely distributed noise. Under certain conditions the optimizationbased models have guarantees on model performance. This paper has sought to build on the success of this research direction, by introducing a Bayesian formalism to this problem. The Bayesian method has several attractive attributes: $(i)$ it is robust to the densely distributed noise and the noise statistics may be inferred based upon the data, with no tuning of hyperparameters; (ii) the framework readily allows the addition of further constraints on the solution, for example in the form of a Markov property on the sparse component, of particular interest for video analysis, and similarly it allows the noise statistics to vary from frame to frame; (iii) for real applications an MCMC-based computational implementation (Gibbs sampler) converges quickly to an accurate mean solution, with computation time commensurate with that of the optimizationbased approach; and (iv) the Bayesian approach also yields "error bars" on the solution, of potential interest for subsequent inferences (classification, tracking, etc.).

There are several directions of interest for future research. For example, in the video examples, a fixed camera was assumed. However, there are clearly many applications for which one may have a moving (e.g., hand-held) video camera. In this case, rather than assuming that the 
background resides on a low-dimensional linear subspace (defined by the low-rank component), it may be more appropriate to assume the background lives on a low-dimensional manifold. The Bayesian formalism may be extended to infer the properties of the low-dimensional manifold (e.g., by a mixture of factor analyzers, rather than by the single factor model associated with the low-rank model).

\section{REFERENCES}

[1] C. Eckart and G. Young, "The approximation of one matrix by another of lower rank," Psychometrika, vol. 1, pp. 211-218, 1936.

[2] J. B. Tenenbaum, V. de Silva, and J. C. Langford, "A global geometric framework for nonlinear dimensionality reduction," Science, vol. 290, pp. 2319-2323, 2000.

[3] J. Verbeek, "Learning nonlinear image manifolds by global alignment of local linear models," IEEE Trans. Pattern Analysis and Machine Intelligence, vol. 28, pp. 1236-1250, 2006.

[4] Netflix, Inc. The Netflix prize. http://www.netflixprize.com/.

[5] I. T. Jolliffe, Principal Component Analysis. Springer-Verlag, 1986.

[6] C. M. Bishop, Pattern Recognition and Machine Learning. S Springer-Verlag, 2006.

[7] J.-F. Cai, E. J. Candès, and Z. Shen, "A singular value thresholding algorithm for matrix completion," SIAM Journal on Optimization, vol. 20, pp. 1956-1982, 2008.

[8] R. Salakhutdinov and A. Mnih, "Probabilistic matrix factorization," in Neural Information Processing Systems (NIPS), 2007.

[9] E. Candès and Y. Plan, "Matrix completion with noise," IEEE Proc., pp. 925 - 936.

[10] J. Wright, Y. Peng, Y. Ma, A. Ganesh, and S. Rao, "Robust principal component analysis: Exact recovery of corrupted low-rank matrices by convex optimization,” in Neural Information Processing Systems (NIPS), 2009.

[11] V. Chandrasekaran, S. Sanghavi, P. A. Parrilo, and A. S. Willsky, "Sparse and low-rank matrix decompositions," in IFAC Symposium on System Identification, 2009.

[12] P. J. Huber and E. M. Ronchetti, Robust Statistics, 2nd ed. Wiley, 2009.

[13] F. D. la Torre and M. J. Black, “A framework for robust subspace learning," International Journal of Computer Vision, vol. 54, pp. 117-142, 2003.

[14] R. Gnanadesikan and J. R. Kettenring, "Robust estimates, residuals, and outlier detection with multiresponse data," Biometrics, vol. 28, pp. 81-124, 1972.

[15] Q. Ke and T. Kanade, "Robust $\ell 1$ norm factorization in the presence of outliers and missing data by alternative convex programming," in IEEE Conf. Computer Vision and Pattern Recognition, 2005.

[16] M. A. Fischler and R. C. Bolles, "Random sample consensus: a paradigm for model fitting with applications to image analysis and automated cartography," Communications of the ACM, vol. 24, pp. 381-385, 1981.

[17] E. J. Candès, X. Li, Y. Ma, and J. Wright, "Robust principal component analysis?" Submitted to Journal of the ACM, 2009.

[18] Z. Zhou, J. Wright, X. Li, E. J. Candès, and Y. Ma, "Stable principal component pursuit," in International Symposium on Information Theory, 2010. 
[19] M. E. Tipping and C. M. Bishop, "Probabilistic principal component analysis," Journal of the Royal Statistical Society Series B, vol. 61, pp. 611-622, 1999.

[20] J. Gao, "Robust $\ell 1$ principal component analysis and its Bayesian variational inference," Neural Computation, vol. 20, pp. 555-578, 2008.

[21] J. Luttinen, A. Ilin, and J. Karhunen, "Bayesian robust PCA for incomplete data," in International Conference on Independent Component Analysis and Signal Separation, 2009.

[22] E. J. Candès and B. Recht, "Exact matrix completion via convex optimization," Foundations of Computational Mathematics, vol. 9, pp. 717-772, 2008.

[23] B. Recht, M. Fazel, and P. A. Parrilo, "Guaranteed minimum-rank solutions of linear matrix equations via nuclear norm minimization," Submitted to SIAM review, 2008.

[24] R. Salakhutdinov and A. Mnih, "Bayesian probabilistic matrix factorization using Markov chain Monte Carlo," in International Conference on Machine Learning, 2008.

[25] Y. J. Lim and Y. W. Teh, "Variational Bayesian approach to movie rating prediction," in KDD Cup and Workshop, 2007.

[26] K. Yu, J. Lafferty, S. Zhu, and Y. Gong, "Large-scale collaborative prediction using a nonparametric random effects model," in International Conference on Machine Learning, 2009.

[27] N. D. Lawrence and R. Urtasun, "Non-lineaar matrix factorization with Gaussian processes," in International Conference on Machine Learning, 2009.

[28] S. Ji, Y. Xue, and L. Carin, "Bayesian compressive sensing," IEEE Transactions on Signal Processing, vol. 56, pp. 23462356, 2008.

[29] M. W. Seeger and H. Nickisch, "Compressed sensing and Bayesian experimental design," in International Conference on Machine Learning, 2008.

[30] D. Wipf, J. Palmer, and B. Rao, "Perspectives on sparse Bayesian learning," in Advances in Neural Information Processing Systems, 2004.

[31] L. He and L. Carin, "Exploiting structure in wavelet-based Bayesian compressive sensing," IEEE Transactions on Signal Processing, vol. 57, pp. 3488-3497, 2009.

[32] M. F. Duarte, M. B. Wakin, and R. G. Baraniuk, "Wavelet-domain compressive signal reconstruction using a hidden Markov tree model," in IEEE International Conference on Acoustics, Speech and Signal Processing (ICASSP), 2008.

[33] M. E. Tipping, "Sparse Bayesian learning and the relevance vector machine," Journal of Machine Learning Research, pp. 211-244, 2001.

[34] P. Hoff, "Simulation of the matrix Bingham-von Mises-Fisher distribution, with applications to multivariate and relational data," J. Comp. Graph. Statistics, 2009.

[35] T. Park and G. Casella, “The Bayesian LASSO,” Journal of the American Statistical Association, vol. 103, pp. 681-686, 2008.

[36] J. Mairal, F. Bach, J. Ponce, and G. Sapiro, “Online dictionary learning for sparse coding," in Proc. International Conference on Machine Learning, 2009.

[37] C. P. Robert and G. Casella, Monte Carlo Statistical Methods, 2nd ed. New York: Springer, 2004.

[38] M. J. Beal, "Variational algorithms for approximate Bayesian inference," Ph.D. dissertation, Gatsby Computational Neuroscience Unit, University College London, 2003.

[39] Z. Lin, M. Chen, L. Wu, and Y. Ma, "The augmented lagrange multiplier method for exact recovery of corrupted low-rank matrices," University of Illinois at Urbana-Champaign, Tech. Rep. UILU-ENG-09-2215, 2009. 